\title{
HAMILTON-JACOBI EQUATIONS WITH STATE CONSTRAINTS
}

\author{
I. CAPUZZO-DOLCETTA AND P.-L. LIONS
}

\begin{abstract}
In the present paper we consider Hamilton-Jacobi equations of the form $H(x, u, \nabla u)=0, x \in \Omega$, where $\Omega$ is a bounded open subset of $R^{n}$, $H$ is a given continuous real-valued function of $(x, s, p) \in \Omega \times R \times R^{n}$ and $\nabla u$ is the gradient of the unknown function $u$. We are interested in particular solutions of the above equation which are required to be supersolutions, in a suitable weak sense, of the same equation up to the boundary of $\Omega$.

This requirement plays the role of a boundary condition. The main motivation for this kind of solution comes from deterministic optimal control and differential games problems with constraints on the state of the system, as well from related questions in constrained geodesics.
\end{abstract}

\section{INTRODUCTION}

This paper is concerned with the study of some particular solutions of general Hamilton-Jacobi equations of the form

$$
H(x, u, \nabla u)=0 \text { in } \Omega
$$

where $\Omega$ is a given domain of $R^{N}$ and $H$-often called the Hamiltonian-is a real-valued function on $\Omega \times R \times R^{N}$ that we will always assume to be at least continuous on $\bar{\Omega} \times R \times R^{N}$. Finally, we will denote the Fréchet differential of $u$ by $\nabla u$.

The solutions of (1) we consider are viscosity solutions "inside $\Omega$ " and viscosity supersolutions (i.e. solutions of $H \geq 0$ ) on $\partial \Omega$. The property that $u$ is a viscosity supersolution on $\partial \Omega$ (see $\S I I$ for precise definitions) plays the role of a boundary condition. For the main facts about viscosity solutions of (1), we refer the reader to M. G. Crandall and P.-L. Lions [8], M. G. Crandall, L. C. Evans and P.-L. Lions [6] (some are briefly recalled in $\S I I$ ). While this paper is mostly self-contained, some knowledge of viscosity solutions is certainly advised.

The main reason why one is interested in such solutions concerns the applications to optimal control theory: roughly speaking, the study of optimal control problems where one restricts the class of controls to those which constrain the

Received by the editors December 15, 1987 and, in revised form, July 18, 1988 .

1980 Mathematics Subject Classification (1985 Revision). Primary 49C05, 49C20.

Part of the contents of this paper have been presented at the Workshop Stochastic Differential Systems, Stochastic Control Theory and Applications held at IMA June 9-19, 1986. 
state of the system inside $\Omega$ (so-called state-constrained problems) leads to viscosity solutions of (1) which are "supersolutions on $\partial \Omega$ " and this is an easy consequence of the dynamic programming argument. For the general relations between optimal control problems, dynamic programming and viscosity solutions we refer to P.-L. Lions [23, 24]. The relations between state-constrained problems and viscosity supersolutions on $\partial \Omega$ are described in M. Soner [32] (see also $\S \mathrm{X}$ ).

In $\S$ II we define precisely solutions of (1) in $\Omega$ which are supersolutions on $\partial \Omega$ and we give a few elementary properties, one of which is of particular interest for optimal control problems. We show that if $u$ is the maximum viscosity subsolution of $(1)$ in $C(\bar{\Omega})$ (i.e. $u \in C(\bar{\Omega})$, is a viscosity solution of (1) $H(x, u, \nabla u) \leq 0$ and any such subsolution is below $u)$, then $u$ is a viscosity solution of $(1)$ and a viscosity supersolution on $\bar{\Omega}$.

In $\S$ III, we present some uniqueness results for such solutions which are obtained by applying the method of proof used by Soner [32] for a particular class of equations.

Next $(\S \mathrm{V})$, we give further uniqueness results by introducing a new assumption based upon the use of distance-like functions. A similar use has been introduced for problems without boundary conditions in M. G. Crandall and P.-L. Lions [9, 10] and for Neumann type boundary conditions and equations like (1) in P.-L. Lions [25]. Here, the distance-like functions have to be carefully chosen.

$\S \S I V$ and VI are devoted to the existence question. It is worth pointing out that restrictions upon $H$ are necessary in view of the counterexamples we give in $\S$ II and that one cannot expect the same generality as in M. G. Crandall and P.-L. Lions $[9,10]$. We first give general existence results ( $§ I V)$ when $H$ grows to $+\infty$ as $|p| \rightarrow \infty$ (at least near $\partial \Omega$ ), while other existence results involving different assumptions (near $\partial \Omega$ ) are proved in $\S \mathrm{VI}$.

In $\S$ VII we present some approximations of the particular solutions of (1) we are interested in, $\S$ VIII is concerned with asymptotic problems related to ergodic state-constrained control problems. In $\S \mathbf{I X}$ we consider an extension of the preceding problems to the case of solutions of (1) which are viscosity supersolutions of (1) on a part of $\partial \Omega$ and satisfy Dirichlet boundary conditions on the complement.

In the last section we apply the results of the preceding sections to various problems of the calculus of variations, optimal control and differential games. In particular, we show various properties such as Lipschitz continuity of the value functions of some control and differential games problems.

\section{ELEMENTARY PROPERTIES}

We first recall a few basic definitions. Let $\Omega$ be a smooth open subset of $R^{N}$ and let $\phi \in C(\bar{\Omega})$. For $x \in \bar{\Omega}$ the subdifferential of $\phi$ at $x$ is the (possibly 
empty) closed convex set defined by

$$
D^{-} \phi(x)=\left\{\xi \in R^{N} \mid \liminf _{y \rightarrow x, y \in \bar{\Omega}} \frac{\phi(y)-\phi(x)-(\xi, y-x)}{|y-x|} \geq 0\right\} .
$$

Similarly, the superdifferential of $\phi$ at $x$-denoted by $D^{+} \phi(x)$-is defined by imposing that the lim sup of the same quantity appearing in (2) is nonpositive. Let us point out explicitly that if $\xi \in D^{-} \phi(x)$ with $x \in \partial \Omega$, then $\xi+c n(x) \in$ $D^{-} \phi(x)$ for all $c \geq 0$, where $n(x)$ is the unit outward normal vector to $\partial \Omega$ at $x$. We may now give the

Definition. A function $u \in C(\bar{\Omega})$ is a viscosity subsolution of (1) on $X$, where $X=\Omega$ or $X=\bar{\Omega}$, if

$$
H(x, u(x), \xi) \leq 0, \quad \forall x \in X, \forall \xi \in D^{+} u(x) .
$$

A function $u \in C(\bar{\Omega})$ is a viscosity supersolution of (1) on $X$ if

$$
H(x, u(x), \xi) \geq 0, \quad \forall x \in X, \forall \xi \in D^{-} u(x) .
$$

Finally, $u$ is a viscosity solution of (1) on $X$ if it is both a subsolution and supersolution on $X$.

This is the usual notion as given in $[8,6]$ and we refer to these papers for the main properties of viscosity solutions. As in $[8,6]$ one can show, using the smoothness of $\Omega$, that the above definition is equivalent to

Equivalent definition. A function $u \in C(\bar{\Omega})$ is a viscosity subsolution of (1) on $X$ if for all $\phi \in C^{1}(\bar{\Omega})$ at any local maximum point $x$ of $u-\phi$ on $X$ the following holds:

$$
H(x, u(x), \nabla \phi(x)) \leq 0 .
$$

A function $u \in C(\bar{\Omega})$ is a viscosity supersolution of (1) on $X$ if for all $\phi \in$ $C^{1}(\bar{\Omega})$ at any local minimum point $x$ of $u-\phi$ on $X$, the following holds:

$$
H(x, u(x), \nabla \phi(x)) \geq 0 .
$$

Remarks. Let us observe explicitly that if $u$ is a viscosity supersolution of (1) on $\bar{\Omega}$ then, in general, $u$ is not a viscosity supersolution in $\bar{\omega}$, where $\omega$ is a subdomain of $\Omega$.

It is worth mentioning also that in R. Jensen [21], M. G. Crandall and R. Newcomb [13], P. E. Souganidis [33] various conditions on $H$ are given which insure that if $u \in C(\bar{\Omega})$ is a viscosity supersolution of (1) in $\Omega$ then $u$ is a viscosity supersolution on $\partial \Omega$ or on some part of $\partial \Omega$. These conditions involve some variations of the following condition:

$$
H(x, t, p+\lambda n(x)) \geq H(x, t, p), \quad \forall x \in \partial \Omega, t \in R, p \in R^{N}, \lambda \geq 0 .
$$

In the rest of this paper we shall be mostly interested in functions $u$ which are subsolutions of (1) in $\Omega$ and supersolutions of (1) in $\bar{\Omega}$, according to the above definition. 
Let us explain now the relations between viscosity supersolutions of (1) in $\bar{\Omega}$ and viscosity supersolutions of the Neumann problem

$$
H(x, u, \nabla u)=0 \quad \text { in } \Omega, \quad \partial u / \partial n=0 \quad \text { on } \partial \Omega .
$$

According to P.-L. Lions [25] (see also B. Perthame and R. Sanders [29]), $u \in$ $C(\bar{\Omega})$ is a viscosity supersolution of (7) if it is a viscosity supersolution of (1) in $\Omega$ and it satisfies

(8) $H(x, u(x), \xi) \geq 0, \quad \forall x \in \partial \Omega, \forall x \in D^{-} u(x)$ such that $(\xi, n(x)) \leq 0$.

Proposition II.1. Let $u \in C(\Omega)$. If $u$ is a viscosity supersolution of (1) on $\bar{\Omega}$, then $u$ is a viscosity supersolution of (7). Conversely, if $u$ is a viscosity supersolution of (7) and if $H(x, u(x), p)$ is a nondecreasing function of $|p|$ for all $x \in \partial \Omega$, then $u$ is a viscosity supersolution of (1) on $\bar{\Omega}$.

Proof. The first statement is obvious since (4) implies (8). Conversely, let $u$ be a viscosity supersolution of (7) and $x \in \partial \Omega$. It is enough to check that

$$
H(x, u(x), \xi) \geq 0, \quad \forall \xi \in D^{-} u(x),(\xi, n(x))>0 .
$$

Let us decompose $\xi$ as

$$
\xi=\xi^{\prime}+(\xi, n(x)) n(x), \quad \text { with }\left(\xi^{\prime}, n(x)\right)=0 .
$$

By an extension lemma proven in [25] we have

$$
H\left(x, u(x), \xi-\lambda_{0} n(x)\right) \geq 0 .
$$

Now, if $2(\xi, n(x)) \geq \lambda_{0}$ then $\left|\xi-\lambda_{0} n(x)\right| \leq|\xi|$ and therefore

$$
H(x, u(x), \xi) \geq H\left(x, u(x), \xi-\lambda_{0} n(x)\right) \geq 0 .
$$

On the other hand, if $0<(\xi, n(x))<\lambda_{0} / 2<\lambda_{0}$, then $\xi^{\prime} \in D^{-} u(x)$ and therefore, taking the assumption on $H$ into account and the fact that $|\xi| \geq\left|\xi^{\prime}\right|$, we obtain

$$
H(x, u(x), \xi) \geq H\left(x, u(x), \xi^{\prime}\right) \geq 0 .
$$

Remark. In order to explain the above result, let us point out that if $u$ is a viscosity supersolution of (1) on $\bar{\Omega}$ which is differentiable on $\bar{\Omega}$, then

$$
D^{-} u(x)=\left\{\xi \in R^{N} \mid \xi=D u(x)+c n(x), c \geq 0\right\}, \quad \text { for } x \in \partial \Omega .
$$

Hence,

$$
H(x, u(x), \nabla u(x))=0, \quad H(x, u(x), \nabla u(x)+c n(x)) \geq 0
$$

for all $c \geq 0$ and $x \in \partial \Omega$. If $H$ is differentiable with respect to $p$, this implies that $u$ satisfies the boundary condition

$$
\frac{\partial H}{\partial n}(x, u(x), \nabla u(x)) \geq 0, \quad x \in \partial \Omega .
$$

Observe finally that if $H$ is nondecreasing with respect to $|p|$ the above yields

$$
2(\nabla u(x), n(x))+c=|\nabla u(x)+c n(x)|^{2}-|\nabla u(x)|^{2} \geq 0 .
$$


Hence, sending $c$ to zero, we obtain

$$
\frac{\partial u}{\partial n}(x) \geq 0, \quad x \in \partial \Omega .
$$

We give now an important observation for the rest of this paper.

Theorem II.1. Let $u \in C(\bar{\Omega})$ be a viscosity subsolution of (1) in $\Omega$. Assume, in addition, that for all viscosity subsolutions $v \in C(\bar{\Omega})$ of $(1)$ in $\Omega$ one has $v \leq u$ in $\bar{\Omega}$. Then $u$ is a viscosity supersolution of (1) in $\bar{\Omega}$.

Proof. We argue by contradiction. Let $\phi \in C^{1}(\Omega), x_{0} \in \bar{\Omega}$; we assume that $u-\phi$ has a global minimum on $\bar{\Omega}$ at $x_{0}$ and that

$$
H\left(x_{0}, u\left(x_{0}\right), \nabla \phi\left(x_{0}\right)\right)<0 \text {. }
$$

We then set $v(x)=u\left(x_{0}\right)+\varepsilon-\left|x-x_{0}\right|^{2}+\phi(x)-\phi\left(x_{0}\right)$ where $\varepsilon>0$ is determined below. Next, let $\delta>0$; we have on $\partial B\left(x_{0}, \delta\right) \cap \bar{\Omega}$

$$
v(x) \leq u(x)+\varepsilon-\delta^{2} \leq u(x)-\varepsilon
$$

as soon as $\delta \geq(2 \varepsilon)^{1 / 2}$. Computing $H(x, v(x), \nabla v(x))$ on $\overline{B\left(x_{0}, \delta\right) \bar{\Omega}}$ we find

$$
\begin{aligned}
H(x, v(x), \nabla v(x)) & =H\left(x, v(x), \nabla \phi(x)-2\left(x-x_{0}\right)\right) \\
& \leq H\left(x_{0}, u\left(x_{0}\right), \nabla \phi\left(x_{0}\right)\right)-\omega(\delta)
\end{aligned}
$$

where $\omega(t) \rightarrow 0$ if $t \rightarrow 0_{+}$. Hence, choosing $\delta$ small enough and then $0 \leq$ $\varepsilon \leq \delta^{2} / 2$, we find

$$
\begin{gathered}
v \in C^{1}(\bar{\Omega}), \quad v\left(x_{0}\right)>u\left(x_{0}\right), \quad v<u \quad \text { on } \partial B\left(x_{0}, \delta\right) \cap \bar{\Omega}, \\
H(x, v(x), \nabla v(x)) \leq 0 \quad \text { in } \overline{B\left(x_{0}, \delta\right)} \cap \bar{\Omega} .
\end{gathered}
$$

Then, we set $w(x)=\max (v(x), u(x))$ in $B\left(x_{0}, \delta\right) \cap \bar{\Omega},=u(x)$ in $B\left(x_{0}, \delta\right)^{c} \cap$ $\bar{\Omega}$. Obviously $w \in C(\bar{\Omega})$ and since $v \leq u$ in a neighborhood of $\partial B\left(x_{0}, \delta\right)$ (in $\bar{\Omega}$ ) we observe that $w$ is a viscosity subsolution of (1) in $\Omega$ : indeed we just have to check that $w$ is a viscosity subsolution of $(1)$ in $B\left(x_{0}, \delta\right) \cap \Omega$ and this follows from the stability of viscosity subsolutions by the operation max. Finally remarking that $w\left(x_{0}\right)>u\left(x_{0}\right)$, we reach a contradiction which proves the theorem.

The next result shows that viscosity supersolutions of (1) on $\bar{\Omega}$ need not exist.

Proposition II.2. Let us assume that for some $x_{0} \in \partial \Omega$ and $\delta>0$ the following holds:

for every $R>0$ there exists $M>0$ such that

$$
H(x, t, p)<0 \text { for }|p|>M,|t| \leq R, x \in \overline{B\left(x_{0}, \delta\right)} \cap \bar{\Omega} .
$$

Then, there is no viscosity supersolution of (1) in $\bar{\Omega}$.

Proof. Let us assume by contradiction that such a viscosity supersolution exists, say $u \in C(\bar{\Omega})$. From (10) it follows that $u$ is Lipschitz continuous on 
$B\left(x_{0}, \delta\right) \cap \Omega$ (see [8]). Let us take $\phi \in C^{1}(\bar{\Omega})$ such that $u-\left.\phi\right|_{\partial \Omega}$ has a global minimum on $\partial \Omega$ at some $x_{1} \in B\left(x_{0}, \delta\right) \cap \partial \Omega$. Then, the function $u-\phi+c d$, where $d(x)=\operatorname{dist}(x, \partial \Omega)$ and $c \geq 0$, has a local minimum at $x_{1}$. Therefore, $\nabla \phi\left(x_{1}\right)+c n\left(x_{1}\right) \in D^{-} u\left(x_{1}\right)$ and consequently

$$
H\left(x_{1}, u\left(x_{1}\right), \nabla \phi\left(x_{1}\right)+c n\left(x_{1}\right)\right) \geq 0 .
$$

For large $c$ this contradicts (10) and the proof is complete.

\section{UNIQUENESS RESULTS}

We present in this section some comparison and uniqueness results for viscosity solutions of

$$
u+H(x, u, \nabla u)=0 \text { in } \Omega
$$

which are supersolutions in $\bar{\Omega}$, as well as for the Cauchy problem

$$
\begin{aligned}
\frac{\partial u}{\partial t}+H(x, t, u, \nabla u) & =0 \quad \text { in } \Omega \times(0, T), \\
u(x, 0) & =u_{0}(x) \text { in } \Omega .
\end{aligned}
$$

We shall always assume that $H \in C\left(\bar{\Omega} \times[0, T] \times R \times R^{N}\right)$ (when dealing with (11) $H$ will be taken independent on $t$ ). The basic assumptions we shall use in this section are:

$$
\begin{array}{r}
H(x, t, s, p) \text { is nondecreasing with respect to } s \\
\text { for all } x \in \bar{\Omega}, t \in[0, t], p \in R^{N}, \\
|H(x, t, s, p)-H(y, t, s, p)| \leq \omega(|x-y|(1+|p|,|s|), \\
\quad \text { for all } x, y \in \bar{\Omega}, t \in[0, T], s \in R, p \in R^{N}, \\
|H(x, t, s, p)-H(x, t, s, q)| \leq \mu(|p-q|,|s|), \\
\text { for } x \in \Gamma_{0}, t \in[0, t], s \in R, p, q \in R^{N} .
\end{array}
$$

Here, $\Gamma_{0}$ is a closed (with respect to $\bar{\Omega}$ ) neighborhood of $\partial \Omega, \omega$ and $\mu$ are local moduli. Let us recall that a modulus is a continuous nondecreasing, nonnegative, subadditive function from $[0, \infty)$ to $(0, \infty)$ such that $\omega(0)=0$. A local modulus $\mu(s, t)$ is a modulus in $s$ for each $t \geq 0$, is continuous in $(s, t)$ and nondecreasing with respect to $t$.

(H4) $\Omega$ is a bounded and starshaped (with respect to the origin) open subset of $R^{N}$ such that

$$
\operatorname{dist}(x, \bar{\Omega}) \geq k \varepsilon, \quad \forall x \in(1+\varepsilon) \partial \Omega, \forall \varepsilon>0,
$$

for some $k>0$.

Assumptions (H1), (H2) appear in $[8,23]$ while $(\mathrm{H} 3)$ and $(\mathrm{H} 4)$ will be specifically needed for the results of this paper.

The comparison results we present below are of different nature. Actually, Theorems III.1, III.2 (and their variants III.3, III.4) make use of assumption 
(H3) and their proofs are based on a combination of arguments in [9] and of M. Soner [32].

For the second class of results (Theorems III.5,6,7) assumption (H3) is not needed while a major role is played by the geometric condition $(\mathrm{H} 4)$.

Theorem III.1. We assume that $\Omega$ is bounded and that $(\mathrm{H} 1)$ holds. Let $u, v \in$ $C(\bar{\Omega})$ be, respectively, a viscosity subsolution of (11) in $\Omega$, and a viscosity supersolution of

$$
v+H(x, v, \nabla v)+f(x)=0 \text { in } \bar{\Omega},
$$

where $f \in C(\bar{\Omega})$. Then, if either (H2), (H3) hold or $u$ is Lipschitz, we have

$$
\max _{\bar{\Omega}}(u-v)^{+} \leq \max _{\bar{\Omega}} f^{+} .
$$

Remarks. (i) Observe that of course no comparison between $u$ and $v$ on $\partial \Omega$ is necessary.

(ii) The main difference between the most general uniqueness results of viscosity solutions and the above one is in the additional assumptions needed: indeed we need either (H3) or we assume that $u$ is Lipschitz.

We do not know if it is enough to assume that $v$ is Lipschitz.

(iii) Theorem II. 1 is, in a sense, the converse of the above result which shows in particular that if $u$ is a viscosity solution in $\Omega$ and a supersolution in $\bar{\Omega}$ then $u$ is the maximum subsolution.

We now turn to (12).

Theorem III.2. We assume that $\Omega$ is bounded and that (H1) holds. Let $u, v \in$ $C(\Omega \times[0, T])$ be, respectively, a viscosity subsolution of (12) and a viscosity supersolution of

$$
\frac{\partial v}{\partial t}+H(x, t, v, \nabla v)+f(x, t)=0 \quad \text { in } \bar{\Omega} \times(0, T)
$$

where $f \in C(\bar{\Omega} \times[0, T])$. Then, if either $(\mathrm{H} 2)$, (H3) hold or $u$ is Lipschitz in $x$ uniformly for $t \in[0, T]$, we have for all $t \in[0, T]$

$$
\max _{\bar{\Omega}}(u-v)^{+}(t) \leq \max _{\bar{\Omega}}(u-v)^{+}(0)+\int_{0}^{t} \max _{\bar{\Omega}} f^{+}(s) d s .
$$

We will only prove Theorem III.1. The proof of Theorem III.2 can be performed then with simple adaptations.

Proof of Theorem III.1. We consider $w_{\varepsilon}(x, y)=u(x)-v(y)-\frac{1}{\varepsilon^{2}}|x+\varepsilon T(x)-y|^{2}$ where $T(x)=\zeta(x) n(x), \zeta \equiv 1$ near $\partial \Omega, \zeta \in C^{1}(\bar{\Omega}), \zeta \equiv 0$ if $x \in \bar{\Omega}-\Gamma_{0}$ and $n(x)=-\nabla d(x)$ (taking $\Gamma_{0}$ small enough so that $d$ is differentiable on $\left.\Gamma_{0}\right)$. If we assume that $\max _{\bar{\Omega}}(u-v)^{+}>\max _{\bar{\Omega}} f^{+}$, then, by the results of $[8$, 23], $\gamma=\max _{\bar{\Omega}}(u-v)^{+}=\max _{\partial \Omega}(u-v)^{+}=(u-v)\left(x_{0}\right)>0$ for some $x_{0} \in \partial \Omega$. Therefore

$$
\max _{\bar{\Omega} \times \bar{\Omega}} w_{\varepsilon}(x, y) \geq w_{\varepsilon}\left(x_{0}-\varepsilon n\left(x_{0}\right), x_{0}\right) \geq \gamma-\omega(\varepsilon)
$$


where $\omega$ is the modulus of continuity of $u$. Now, if $(\bar{x}, \bar{y}) \in \bar{\Omega} \times \bar{\Omega}$ is a maximum point of $w_{\varepsilon}$ we find

$$
\max _{\bar{\Omega} \times \bar{\Omega}} w_{\varepsilon}(x, y)=w_{\varepsilon}(\bar{x}, \bar{y}) \leq \gamma+\omega(|\bar{x}-\bar{y}|)-\frac{1}{\varepsilon^{2}}|\bar{x}+\varepsilon T(\bar{x})-\bar{y}|^{2} .
$$

Hence, combining (17) and (18),

$$
|\bar{x}+\varepsilon T(\bar{x})-\bar{y}|^{2} \leq \varepsilon^{2} \omega(|\bar{x}-\bar{y}|)+\omega(\varepsilon) \varepsilon^{2} .
$$

This implies easily $|\bar{x}-\bar{y}| \leq C \varepsilon$ and therefore

$$
|\bar{x}+\varepsilon T(\bar{x})-\bar{y}| \leq \varepsilon \delta(\varepsilon),
$$

where $\delta(t) \rightarrow 0$ as $t \rightarrow 0_{+}$. Now, in view of the properties of $T$, this yields $\bar{x} \in \Omega$ for $\varepsilon$ small enough.

Applying the definitions of viscosity sub and supersolutions we now obtain

$$
\begin{aligned}
& u(\bar{x})+H\left(\bar{x}, u(\bar{x}), \frac{2}{\varepsilon^{2}}(I+\varepsilon \nabla T(\bar{x}))(\bar{x}+\varepsilon T(\bar{x})-\bar{y})\right) \leq 0, \\
& v(\bar{y})+H\left(\bar{y}, v(\bar{y}), \frac{2}{\varepsilon^{2}}(\bar{x}+\varepsilon T(\bar{x})-\bar{y})\right)+f(\bar{y}) \geq 0 .
\end{aligned}
$$

Then, if $\bar{x} \notin \Gamma_{0}, T(\bar{x})=0$ and standard arguments of $[8,6]$ apply and we conclude subtracting the above inequality and using (H2), (19). If $\bar{x} \in \Gamma_{0}$, we first observe that in view of (19)

$$
\left|\frac{2}{\varepsilon^{2}}(\varepsilon \nabla T(\bar{x}))(\bar{x}+\varepsilon T(\bar{x})-\bar{y})\right| \leq C \delta(\varepsilon) .
$$

Using (H3) we deduce from the above inequalities

$$
u(\bar{x})-v(\bar{y})+H\left(\bar{x}, u(\bar{x}), \frac{2}{\varepsilon^{2}}(\bar{x}+\varepsilon T(\bar{x})-\bar{y})\right) \leq \kappa(\varepsilon) \rightarrow 0 \quad \text { as } \varepsilon \rightarrow 0_{+} .
$$

If (H2) holds, the remainder of the argument is standard (see $[8,6])$. If $u$ is Lipschitz, we use the fact that this implies that

$$
\left|\frac{2}{\varepsilon^{2}}(I+\varepsilon \nabla T(\bar{x}))(\bar{x}+\varepsilon T(\bar{x})-\bar{y})\right| \leq C
$$

where $C$ is a bound for $|\nabla u|$. This yields

$$
\left|\frac{2}{\varepsilon^{2}}(\bar{x}+\varepsilon T(\bar{x})-\bar{y})\right| \leq C+C \delta(\varepsilon)
$$

and we conclude as usual.

Remark. We would like to point out that it is possible to relax somehow (H2) by assuming instead

$$
\begin{aligned}
& H\left(x, t, s, \lambda \nabla_{x} d(x, y)\right)-H\left(y, t, s,-\lambda \nabla_{y} d(x, y)\right) \\
& \geq-\omega(\lambda d(x, y)+d(x, y),|s|) \\
& \quad \text { for all } x \neq y \in \bar{\Omega}, t \in[0, T], s \in R^{N}, 0 \leq \lambda,
\end{aligned}
$$


for some local modulus $\omega$, where $d(x, y)$ is defined on a neighborhood of $\bar{\Omega} \times \bar{\Omega}$, is Lipschitz, differentiable with respect to $x$ for $x \neq y$ and with respect to $y$ for $y \neq x, d(x, y) \geq|x-y|, d(x, x)=0$ for all $x, y$. In addition, we need to assume

$$
|H(x, t, s, p)-H(y, t, s, p)| \leq \omega(|x-y|(1+|p|),|s|)
$$

for all $x, y$ in a neighborhood of $\partial \Omega, t \in[0, T], s \in R, p \in R^{N}$,

$$
\begin{aligned}
& \left|\nabla_{x} d^{2}(x, y)+\nabla_{y} d^{2}(x, y)\right| \leq C d^{2}(x, y), \\
& \left|\nabla_{x} d^{2}\left(x_{1}, y\right)-\nabla_{x} d^{2}\left(x_{2}, y\right)\right| \leq C\left|x_{1}-x_{2}\right|
\end{aligned}
$$

for all $x_{1}, x_{2}, x, y$ in a neighborhood of $\partial \Omega$, for some $C \geq 0$.

We now conclude this section by briefly mentioning the case when $\Omega$ is unbounded and we work with uniformly continuous functions. We need some additional assumptions:

$$
\begin{aligned}
& H(x, t, s, p)-H(x, t, s,+\lambda \nabla \nu(x)) \leq C_{R} \\
& \quad \text { for } x \in \bar{\Omega}, t \in[0, T], s \in R, p \in B_{R}, 0 \leq \lambda \leq R,
\end{aligned}
$$

for some $C_{R} \geq 0$ where $R>0$ is arbitrary.

There is a local modulus $\sigma$ such that

$$
\begin{aligned}
& H(x, t, s, p)-H(x, t, s, p+\lambda D \mu(x)) \leq \sigma(\lambda,|p|) \\
& \quad \text { for } x \in \bar{\Omega}, t \in[0, T], s \in R, p \in R^{N}, 0 \leq \lambda \leq 1,
\end{aligned}
$$

where $\nu, \mu$ are Lipschitz, nonnegative and differentiable, $\mu \rightarrow+\infty$ as $|x| \rightarrow$ $\infty, \nu(x) \geq|x|$ for $x$ large.

Finally, we will use some variants of (H2):

$$
\begin{gathered}
\text { (H2s) } \begin{array}{r}
|H(x, t, s, p)-H(y, t, s, p)| \leq \omega(|x-y|(1+|p|)) \\
\text { for } x, y \in \bar{\Omega}, t \in[0, T], s \in R, p \in R^{N}, \\
\text { (H2w) }|H(x, t, s, p)-H(y, t, s, p)| \leq m(|x-y|,|p|)
\end{array} \\
\quad \text { for } x, y \in \bar{\Omega}, t \in[0,1], s \in R, p \in R^{N}, \\
\left(\mathrm{H} 2 \mathrm{w}^{\prime}\right) \quad|H(x, t, s, p)-H(y, t, s, p)| \leq m(|x-y|,|p|+|s|) \\
\\
\quad \text { for } x, y \in \bar{\Omega}, t \in[0,1], s \in R, p \in R^{N},
\end{gathered}
$$

where $\omega$ is a modulus and $m, \sigma$ are local moduli.

Theorem III.3. Assume that $(\mathrm{H} 1)$ holds. Let $u, v \in C(\Omega)$ be respectively a viscosity subsolution of (11) in $\Omega$ and a viscosity supersolution of (13) in $\bar{\Omega}$ where $f \in C_{b}(\bar{\Omega})$.

We assume one of the following four sets of assumptions: (i) $u, v$ are uniformly continuous on $\bar{\Omega}$ and (22), (23), (H2s), (H3) hold, (ii) $u$ is Lipschitz on $\bar{\Omega}$ and (22), (23), (H2w) hold, (iii) $u, v$, are bounded uniformly continuous on $\bar{\Omega}$ and (23), (H2), (H3) hold; (iv) $u, v$ are bounded on $\bar{\Omega}, u$ is Lipschitz on $\bar{\Omega}$ and (23), (H2w') hold. Then (14) holds. 
Theorem III.4. Assume that $(\mathrm{H} 1)$ holds. Let $u, v \in B U C\left(\left(B_{R} \cap \bar{\Omega}\right) \times[0, T]\right)$ $(\forall R<\infty)$ be respectively a viscosity subsolution of (12) and a viscosity super solution of (15) where $f \in C_{b}(\bar{\Omega} \times[0, T])$. We assume one of the following sets of conditions: (i) $u, v$ are uniformly continuous in $x$ uniformly in $t \in$ [0,T] and (22), (23), (H2s), (H3) hold; (ii) $u$ is Lipschitz in $x$ uniformly in $t \in[0, T]$ and (22), (23), ( $\mathrm{H} 2 \mathrm{w})$ hold; (iii) $u, v$ are bounded uniformly continuous in $x$ uniformly in $t \in[0, T]$ and (23), (H2), (H3) hold; (iv) $u, v$ are bounded, $u$ is Lipschitz in $x$ uniformly in $t \in[0, T]$ and $(23),\left(\mathrm{H} 2 \mathrm{w}^{\prime}\right)$ hold. Then (16) holds.

Since the proof of these results is, as above, a combination of M. Soner's method and the arguments of $[9,10]$, we will skip them. Let us mention also that some uniqueness results for unbounded $\Omega$ can be proved by combining the methods of M. G. Crandall and P.-L. Lions [11] and of M. Soner [32], provided suitable conditions at infinity are satisfied.

Let us consider now the case where $\Omega$ is star-shaped and assume for simplicity that $H$ does not depend on $s$.

Theorem III.5. Let $u, v \in C(\bar{\Omega})$ be, respectively, a viscosity subsolution of

$$
u+H(x, \nabla u)=0 \text { in } \Omega
$$

and a viscosity supersolution of

$$
v+H(x, \nabla v)+f=0 \quad \text { in } \bar{\Omega}
$$

where $f \in C(\bar{\Omega})$. Then, if $(\mathrm{H} 2),(\mathrm{H} 4)$ hold, we have

$$
\max _{\bar{\Omega}}(u-v)^{+} \leq \max _{\bar{\Omega}} f^{+} .
$$

Proof. Let $x_{0} \in \partial \Omega$ be a strict maximum point for $u-v$ on $\bar{\Omega}$ and $\left(x_{\varepsilon}, y_{\varepsilon}\right) \in$ $(1+\varepsilon) \bar{\Omega} \times \bar{\Omega}$ be a maximum point for

$$
u_{\varepsilon}(x)-v(y)-\frac{1}{2 \varepsilon^{2}}|x-y|^{2}, \quad \varepsilon>0,
$$

where

$$
u_{\varepsilon}(x)=(1+\varepsilon) u\left(\frac{x}{1+\varepsilon}\right), \quad x \in(1+\varepsilon) \bar{\Omega} .
$$

Since $\Omega$ is starshaped, $x_{0} \in(1+\varepsilon) \bar{\Omega}$ and therefore

$$
u_{\varepsilon}\left(x_{\varepsilon}\right)-v\left(y_{\varepsilon}\right)-\frac{1}{2 \varepsilon^{2}}\left|x_{\varepsilon}-y_{\varepsilon}\right|^{2} \geq u_{\varepsilon}\left(x_{0}\right)-v\left(x_{0}\right) .
$$

This yields $\left|x_{\varepsilon}-y_{\varepsilon}\right| \rightarrow 0$ as $\varepsilon \rightarrow 0$. Therefore, $x_{\varepsilon}$ and $y_{\varepsilon}$ have a common limit $\tilde{x}$ as $\varepsilon \rightarrow 0$. From (27) we deduce that

$$
\limsup _{\varepsilon \rightarrow 0} \frac{\left|x_{\varepsilon}-y_{\varepsilon}\right|^{2}}{2 \varepsilon^{2}} \leq \lim _{\varepsilon \rightarrow 0}\left[u_{\varepsilon}\left(x_{\varepsilon}\right)-v\left(y_{\varepsilon}\right)-u_{\varepsilon}\left(x_{0}\right)+v\left(x_{0}\right)\right] \leq 0
$$

so that $\operatorname{dist}\left(x_{\varepsilon}, \bar{\Omega}\right)=O(\varepsilon)$. By assumption $(\mathrm{H} 4)$ then $x_{\varepsilon} \in(1+\varepsilon) \Omega$. 
Now, $u_{\varepsilon}$ is a viscosity subsolution of

$$
\frac{1}{1+\varepsilon} u_{\varepsilon}+H\left(\frac{x}{1+\varepsilon}, \nabla u_{\varepsilon}\right)=0 \text { in } \Omega \text {. }
$$

In the standard way we obtain

$$
\frac{1}{1+\varepsilon} u_{\varepsilon}\left(x_{\varepsilon}\right)+H\left(\frac{x_{\varepsilon}}{1+\varepsilon}, \frac{x_{\varepsilon}-y_{\varepsilon}}{\varepsilon^{2}}\right) \leq 0
$$

and, from (25),

$$
v\left(y_{\varepsilon}\right)+H\left(y_{\varepsilon}, \frac{x_{\varepsilon}-y_{\varepsilon}}{\varepsilon^{2}}\right)+f\left(y_{\varepsilon}\right) \geq 0 .
$$

By subtraction and using $(\mathrm{H} 2)$ we find

$$
\frac{1}{1+\varepsilon} u_{\varepsilon}\left(x_{\varepsilon}\right)-v\left(y_{\varepsilon}\right) \leq \omega\left(\frac{\left|x_{\varepsilon}-y_{\varepsilon}\right|+\varepsilon\left|y_{\varepsilon}\right|}{1+\varepsilon}\left(1+\frac{\left|x_{\varepsilon}-y_{\varepsilon}\right|}{\varepsilon^{2}}\right)\right)+\underset{\bar{\Omega}}{\operatorname{Max}} f^{+}
$$

and (26) follows passing to the limit as $\varepsilon \rightarrow 0$ in the above inequality.

For the next result we observe that since $\Omega$ is assumed to be smooth, the following property holds:

For all $x \in \partial \Omega$ there exists a neighborhood $Q$ (relative to $\Omega$ ) satisfying (H4).

Theorem III.6. Let $u, v \in C(\Omega)$ be, respectively, a viscosity subsolution of (24) and a supersolution of (25) .

If we assume that (28), ( $\mathrm{H} 2)$ hold and

$$
p \rightarrow H(x, p) \text { is convex for all } x \in \bar{\Omega},
$$

then, $\max _{\bar{\Omega}}(u-v)^{+} \leq \max _{\bar{\Omega}} f^{+}$.

Proof. Let $x^{\theta}$ be a maximum point of $\theta u-v$ on $\bar{\Omega}, 0<\theta<1$. It is easy to check, by the same arguments used in the proof of Theorem III.5, that if $\left(x_{\varepsilon}^{\theta}, y_{\varepsilon}^{\theta}\right)$ is a maximum point for

$$
\theta u(x)-v(y)-\frac{1}{2 \varepsilon^{2}}|x-y|^{2}
$$

then

$$
\begin{gathered}
\frac{\left|x_{\varepsilon}^{\theta}-y_{\varepsilon}^{\theta}\right|^{2}}{\varepsilon^{2}} \rightarrow 0 \quad \text { as } \varepsilon \rightarrow 0, \\
\lim _{\varepsilon \rightarrow 0} x_{\varepsilon}^{\theta}=\lim _{\varepsilon \rightarrow 0} y_{\varepsilon}^{\theta}=x^{\theta} .
\end{gathered}
$$

Therefore, if $x^{\theta} \in \Omega$ then $\left(x_{\varepsilon}^{\theta}, y_{\varepsilon}^{\theta}\right) \in \Omega \times \Omega$ for small $\varepsilon>0$. Observe now that (29) implies that $\theta u$ is a viscosity subsolution of

$$
\theta u+H(x, \nabla \theta u) \leq(1-\theta) H(x, 0) \quad \text { in } \Omega .
$$

Hence, by the usual technique,

$$
\theta u\left(x_{\varepsilon}^{\theta}\right)-v\left(y_{\varepsilon}^{\theta}\right) \leq \omega\left(\left|x_{\varepsilon}^{\theta}-y_{\varepsilon}^{\theta}\right|\left(1+\frac{\left|x_{\varepsilon}^{\theta}-y_{\varepsilon}^{\theta}\right|}{\varepsilon^{2}}\right)\right)+(1-\theta) H\left(x_{\varepsilon}^{\theta}, 0\right)+\underset{\bar{\Omega}}{\operatorname{Max}} f^{+}
$$


and the statement follows by letting $\varepsilon \rightarrow 0$ and $\theta \rightarrow 1$. Assume now that $x^{\theta} \in \partial \Omega$ and consider

$$
\tilde{u}=\theta u-(1-\theta) \frac{\left|x-x^{\theta}\right|^{2}}{2} .
$$

It is straightforward to check that $\tilde{u}$ is a viscosity subsolution of

$$
\tilde{u}+H(x, \nabla \tilde{u}) \leq(1-\theta)\left(H\left(x,-\left(x-x^{\theta}\right)\right)-\frac{\left|x-x^{\theta}\right|^{2}}{2}\right) \quad \text { in } \Omega
$$

and that $x^{\theta}$ is a strict maximum point for $\tilde{u}-v$. Let us take now a neighborhood of $x^{\theta}$, say $Q=B\left(x^{\theta}, \delta\right) \cap \Omega$ satisfying (28) and define $\tilde{u}_{\varepsilon}$ on $(1+\varepsilon) \bar{Q}$ by

$$
\tilde{u}_{\varepsilon}(x)=\frac{1}{1+\varepsilon} \tilde{u}\left(\frac{x}{1+\varepsilon}\right) .
$$

As in the proof of Theorem III.5, one can show that if $\left(x^{\theta}, y^{\theta}\right)$ is a maximum for $\tilde{u}_{\varepsilon}(x)-v(y)-|x-y|^{2} / 2 \varepsilon^{2}$, then

$$
\frac{\left|x_{\varepsilon}^{\theta}-y_{\varepsilon}^{\theta}\right|^{2}}{\varepsilon^{2}} \rightarrow 0
$$

and, by $(28), x_{\varepsilon}^{\theta} \in(1+\varepsilon) Q$.

The usual method then applies yielding

$$
\begin{aligned}
\frac{1}{1+\varepsilon} \tilde{u}_{\varepsilon}\left(x_{\varepsilon}^{\theta}\right)-v\left(y_{\varepsilon}^{\theta}\right) \leq & \omega\left(\frac{\left|x_{\varepsilon}^{\theta}-y_{\varepsilon}^{\theta}\right|+\varepsilon\left|y_{\varepsilon}^{\theta}\right|}{1+\varepsilon}\left(1+\frac{\left|x_{\varepsilon}^{\theta}-y_{\varepsilon}^{\theta}\right|}{\varepsilon^{2}}\right)\right) \\
& +\operatorname{Max}_{\bar{\Omega}} f^{+}+C(1-\theta),
\end{aligned}
$$

where $C$ is a bound for the right term in (31).

The statement now follows by taking limits in (32) as $\varepsilon \rightarrow 0$ and $\theta \rightarrow 1$.

In the next result the convexity assumption (29) is substituted by

$$
H \text { is uniformly continuous in } p \text { with a modulus independent }
$$
on $x$, for $x$ in a neighborhood of $\partial \Omega$.

Theorem III.7. Let $u, v \in C(\Omega)$ be, respectively, a viscosity subsolution of (24) and a supersolution of $(25)$. If $(28),(33)$ and $(\mathrm{H} 2)$ hold, then

$$
\max _{\bar{\Omega}}(u-v)^{+} \leq \max _{\bar{\Omega}} f^{+} .
$$

Proof. Let $x_{0} \in \partial \Omega$ be a maximum point of $u-v$ and define $w_{\delta}=u-v-$ $\frac{\delta}{2}\left|x-x_{0}\right|^{2}, \delta>0$. Obviously $x_{0}$ is a strict maximum point for $w_{\delta}$. From (33) it follows that $u_{\delta}=u-\frac{\delta}{2}\left|x-x_{0}\right|^{2}$ is a viscosity subsolution of

$$
u_{\delta}+H\left(x, \nabla u_{\delta}\right) \leq C \delta+\mu(\delta)
$$

where $\mu$ is a modulus for $H$, in a neighborhood $Q$ of $x_{0}$. At this point we are in the same situation as in Theorem III.6 and therefore we omit the rest of the proof. 
Remarks. (i) The comparison inequality (26) can also be proved under assumptions (28), (H2) and the following condition: there exists $\varepsilon_{n} \rightarrow 0$ and $\psi_{n} \in C^{1}(\bar{\Omega})$ with $\psi_{n} \rightarrow 1$ in $C^{1}(\bar{\Omega})$ such that

$$
\lim _{n \rightarrow+\infty} \sup _{\substack{|t| \leq R,|q| \leq \varepsilon_{n}}}\left\{H\left(x, \psi_{n} p+t \nabla \psi_{n}+q\right)-H(x, p)\right\}^{+}=0 .
$$

The proof of this claim is similar to those of Theorems III.6 and III.7. The key observation is that $u_{n}=u / \psi_{n}$ and $\tilde{u}_{n}=u_{n}-\varepsilon_{n}\left|x-x_{n}\right|^{2} / L$, where $x_{n}$ is the maximum point of $u_{n}-v$, are viscosity subsolutions of

$$
u_{n}+H\left(x, \nabla u_{n}\right) \leq \varepsilon_{n}+\delta_{n}
$$

with $\delta_{n} \rightarrow 0$ as $n \rightarrow+\infty$, a consequence of the above assumption.

(ii) Similar comparison results can be proved for the Cauchy problem (12) with simple modifications.

(iii) It is not difficult to analyse the role of the assumption that $\Omega$ is smooth in the preceding proof. This allows us to extend the previous results to more general domains (like, for instance, convex domains).

(iv) Since (H3) is equivalent to (33), we see that Theorem III.7 does not really cover new cases. However, the proof is quite different and yields various possible extensions that we skip here.

\section{EXISTENCE RESUlTS FOR COERCIVE HAMILTONIANS}

To simplify the presentation we will assume that $\Omega$ is a smooth, bounded open set in $R^{N}$. We consider existence results for the stationary problem (11) or for the Cauchy problem (12). We will use the following assumptions:

or

$$
\begin{aligned}
& H(x, t, s, p) \rightarrow+\infty \text { as }|p| \rightarrow \infty, \text { uniformly for } x \in \bar{\Omega}, t \in \\
& {[0, T], s \geq-R, \text { for all } R \geq 0,}
\end{aligned}
$$

(35) $\quad H(x, t, s, p) \rightarrow \infty$ as $|p| \rightarrow \infty$, uniformly for $x$ a neighborhood of $\partial \Omega t \in[0, T], s \geq-R$, for all $R \geq 0$,

$$
\begin{aligned}
& H\left(x, t_{1}, s, p\right)-H\left(x, t_{2}, s, p\right) \leq m\left(t_{1}-t_{2}\right) \text { for all } x \in \bar{\Omega}, \\
& 0 \leq t_{2} \leq t_{1} \leq T, s \in R, p \in R^{N},
\end{aligned}
$$

for some modulus $m$. We may now state

Theorem IV.1 (The stationary case). Assume (H1), and either (34) or (35) and (H2). Then there exists a unique viscosity solution $u \in C(\bar{\Omega})$ of (11) which is a viscosity supersolution on $\bar{\Omega}$.

Theorem IV.2 (The Cauchy problem). Assume (H1), (36) and either (34) or (35) and $(\mathrm{H} 2)$. Let $u_{0} \in C(\bar{\Omega})$. Then there exists a viscosity solution $u \in$ $C(\bar{\Omega} \times[0, T])$ of (12) which is a viscosity supersolution on $\bar{\Omega} \times(0, T)$ and which satisfies $u(x, 0)=u_{0}(x)$ on $\bar{\Omega}$.

Proofs. The uniqueness part in Theorem IV.1 comes from the fact that (34) (or (35)) implies that any viscosity solution of (11) is Lipschitz (near $\partial \Omega$ ) (see [8, 
23]) and the results of $\S I I I$. The existence part in Theorem IV.2 is an adaptation of a general observation of G. Barles: indeed by the resuits [2] one may use (36) to approximate uniformly $H$ by a sequence of Hamiltonians satisfying

$$
\begin{aligned}
& H\left(x, t_{1}, s, p\right)-H\left(x, t_{2}, s, p\right) \leq C\left(t_{1}-t_{2}\right) \text { for all } x \in \bar{\Omega}, \\
& 0 \leq t_{2} \leq t_{1} \leq T, s \in R, \text { for } \in R^{N}
\end{aligned}
$$

for some $C \geq 0$. Then the existence part will give the existence of a solution Lipschitz in $(x, t)$ if (34) holds (or Lipschitz in $(x, t)$ near $\partial \Omega$ if (35) holds). By simple comparison arguments we see it is enough to prove the existence part of Theorem V.2 if $H$ satisfies $(36)^{\prime}$ and if $u \in W^{1, \infty}(\Omega)$ with the Lipschitz regularity mentioned.

We now prove the above statements by a penalty argument. We first extend $H$ to $R^{N} \times[0, T] \times R^{N}$ in such a way that it satisfies the same assumptions on $R^{N}$ and $H \in B U C\left(R^{N} \times[0, T] \times[-R,+R] \times \bar{B}_{R}\right)$ for all $R<\infty$. We also extend $u_{0}$ to $R^{N}$ in $W^{1, \infty}\left(R^{N}\right)$, and we consider $p \in B U C\left(R^{N}\right)$ such that $p \equiv 0$ in $\bar{\Omega}$ and such that $\forall \varepsilon>0, \exists \delta>0, p(x) \geq \delta$ if $\operatorname{dist}(x, \bar{\Omega}) \geq \varepsilon$, $x \in R^{N}-\bar{\Omega}$. We then introduce the problems

$$
u_{\varepsilon}+H\left(x, u_{\varepsilon}, \nabla u_{\varepsilon}\right)=\frac{1}{\varepsilon} p \quad \text { in } R^{N}
$$

and

$$
\begin{aligned}
& \frac{\partial u_{\varepsilon}}{\partial t}+H\left(x, t, u_{\varepsilon}, \nabla u_{\varepsilon}\right)=\frac{1}{\varepsilon} p \quad \text { in } R^{N} \times(0, T), \\
& \left.u_{\varepsilon}\right|_{t=0}=u_{0} \quad \text { in } R^{N} .
\end{aligned}
$$

By the results of P.-L. Lions [23], G. Barles [1, 2], M. G. Crandall and P.-L. Lions $[9,10]$ we know there exist viscosity solutions $u_{\varepsilon} \in B U C\left(R^{N}\right)$ (resp. $B U C\left(R^{N} \times[0, T]\right)$ of (37) (resp. (38))). Furthermore, $u_{\varepsilon_{1}} \leq u_{\varepsilon_{2}}$ if $0<\varepsilon_{2} \leq \varepsilon_{1}$ by standard comparison results on viscosity solutions. Furthermore, there exists a constant $C \geq 0$ such that

$$
u_{\varepsilon} \geq-C, \quad \text { for all } \varepsilon>0 .
$$

In addition, $\left(39^{\prime}\right)$ implies that we have

$$
\frac{\partial u}{\partial t} \leq-C \text { in }{ }^{\prime}\left(R^{N} \times(0, T)\right) \text {, for all } \varepsilon>0
$$

in the case of the Cauchy problem (38). Now (39), (40) imply that $\nabla u_{\varepsilon}$ is bounded on $\Omega$ (resp. $\Omega \times(0, T)$ ) uniformly in $\varepsilon$, or in a neighborhood of $\partial \Omega$ if we only assume (35). But then, using bounds in the equations we find that $u_{\varepsilon}$ is also bounded on $\Omega$ (resp. $\Omega \times(0, T)$ ) uniformly in $\varepsilon$ (in a neighborhood of $\partial \Omega$ if (35) holds). If (34) holds, $u_{\varepsilon}$ is bounded in $W^{1, \infty}$ and thus $u_{\varepsilon}$ converges to some $u \in W^{1, \infty}$ which is a viscosity solution of (11) (resp. (12)). If (35) holds, $u_{\varepsilon}$ is bounded in $W^{1, \infty}$ in a neighborhood of $\partial \Omega$ and we deduce from the methods of M. G. Crandall and P.-L. Lions [10] that 
$u_{\varepsilon}$ has a fixed modulus of continuity on $\bar{\Omega}$ (resp. $\left.\bar{\Omega} \times[0, T]\right)$ independent of $\varepsilon$. Therefore, again $u_{\varepsilon}$ converges uniformly to some $u$ which is Lipschitz near $\partial \Omega$ and which is a viscosity solution of (11) (resp. (12)).

To conclude, we have to prove that $u$ is a viscosity supersolution of (11) (resp. (12)) on $\bar{\Omega}$ (resp. on $\bar{\Omega} \times(0, T)$ ). The proof being the same for (11) and (12), we will give the proof only in the case of the stationary problem (11). Let $\phi \in C^{1}(\bar{\Omega})$ and let $x_{0} \in \partial \Omega$ be a minimum point of $u-\phi$. We may assume that the minimum is strict. Thanks to (39) we can extend $\phi$ to $R^{N}$ in $C^{1}\left(R^{N}\right)$ so that

$$
\phi(y)-\phi\left(x_{0}\right)+1 \leq u_{\varepsilon}(y)-u\left(x_{0}\right)
$$

if $\operatorname{dist}(y, \bar{\Omega})=1$. Next, let $x_{\varepsilon}$ be a minimum point of $u_{\varepsilon}-\phi$ over the set $\left\{x \in R^{N}, \operatorname{dist}(x, \bar{\Omega}) \leq 1\right\}$. By the above choice of $\phi$ we see that $x_{\varepsilon}$ is a local minimum point of $u_{\varepsilon}-\phi$ for $\varepsilon$ is small enough. Of course we may assume that $x_{\varepsilon} \rightarrow \bar{x}$ as $\varepsilon$ goes to 0 (taking a subsequence if necessary). We first claim that $\bar{x} \in \bar{\Omega}$. Indeed, we have

$$
u_{\varepsilon}\left(x_{\varepsilon}\right)+H\left(x_{\varepsilon}, u_{\varepsilon}, \nabla \phi\left(x_{\varepsilon}\right)\right) \geq \frac{1}{\varepsilon} p\left(x_{\varepsilon}\right)
$$

and since $u_{\varepsilon}\left(x_{\varepsilon}\right) \leq u_{\varepsilon}\left(x_{0}\right)+\phi(\bar{x})-\phi\left(x_{0}\right) \leq C$ we finally obtain $p\left(x_{\varepsilon}\right) \leq C \varepsilon$ and thus $p(\bar{x})=0$, i.e. $\bar{x} \in \bar{\Omega}$. We next claim that $\bar{x}=x_{0}$ and $u_{\varepsilon}\left(x_{\varepsilon}\right) \rightarrow u\left(x_{0}\right)$. Indeed, on the open sets $\{x / p(x)<C \varepsilon\}$ for any constant $C \geq 0$ we deduce as above that the functions $u_{\varepsilon}$ are bounded in Lipschitz norm. Thus $u_{\varepsilon}\left(x_{\varepsilon}\right) \rightarrow$ $u\left(x_{0}\right)$ as $\varepsilon \rightarrow 0$. If $\bar{x} \neq x_{0}$, this would imply

$$
\begin{aligned}
u\left(x_{0}\right)-\phi\left(x_{0}\right) & <u(\bar{x})-\phi(\bar{x})=\lim _{\varepsilon \rightarrow 0} u_{\varepsilon}\left(x_{\varepsilon}\right)-\phi\left(x_{\varepsilon}\right) \\
& \leq \lim _{\varepsilon \rightarrow 0} u_{\varepsilon}\left(x_{0}\right)-\phi\left(x_{0}\right)=u\left(x_{0}\right)-\phi\left(x_{0}\right)
\end{aligned}
$$

and the contradiction proves our claim. We conclude then passing to the limit in $(41)$ as $\varepsilon$ goes to 0 .

Remark. Of course we do not know in Theorem IV.2 if the solution is unique. Observe, however, that the way we built the solution shows that $u$ is the minimum viscosity supersolution $v$ of $(12)$ on $\bar{\Omega} \times(0, T)$ such that $\left.v\right|_{t=0} \geq u_{0}$ on $\bar{\Omega}$. Another observation consists in the fact that the solution built $u$ is the limit of the Lipschitz function $u_{\varepsilon}$ on $\bar{\Omega} \times[0, T]$ which are viscosity subsolutions of

$$
\left.\frac{\partial u_{\varepsilon}}{\partial t}+H\left(x, t, u_{\varepsilon}, \nabla u_{\varepsilon}\right) \leq \varepsilon \text { in } \Omega \times\right] 0, T[
$$

and supersolutions of

$$
\left.\frac{\partial u_{\varepsilon}}{\partial t}+H\left(x, t, u \varepsilon, \nabla u_{\varepsilon}\right) \geq-\varepsilon \text { in } \bar{\Omega} \times\right] 0, T[,
$$

and which satisfy $\left\|u_{\varepsilon}(\cdot, 0)-u_{0}(\cdot)\right\|_{\infty} \leq \varepsilon$, and $u$ is the unique solution in that class. 


\section{FURTHER UNIQUENESS RESULTS}

We want to illustrate in this section the use of a new assumption involving some appropriate distance-like function $d(x, y)$. This new assumption enables us to prove some additional uniqueness results and it will play an important role in existence theory (see $\S \mathrm{VI}$ ). To simplify the presentation, we will consider here only the case when $\Omega$ is bounded.

We will assume that there exists a function $d(x, y)$ on $\bar{\Omega} \times \bar{\Omega}$ which is Lipschitz continuous and satisfies $d(x, y) \geq|x-y|, d(x, x)=0$ for all $x, y \in \bar{\Omega}$ and such that there exist a local modulus $\omega$, a positive constant $\lambda_{0} \geq 1$ for which we have

$$
\begin{aligned}
& \lambda d(x, y)=z(x, y) \text { is a viscosity supersolution on } \bar{\Omega} \times \Omega \text { of } \\
& \inf \left\{H\left(x, t, s, \nabla_{x} z\right)-H\left(y, t, s,-\nabla_{y} z\right)+\omega(\lambda|x-y|+|-y|,|s|),\right. \\
& t \in[0, T], s \in R\} \geq 0 \text { on } \bar{\Omega} \times \Omega, \text { for all } \lambda \geq \lambda_{0} .
\end{aligned}
$$

As it can be seen from the considerations in $[10,7]$, the main new fact lies in the possibility of having a supersolution on $\partial \Omega \times \Omega$ and this may be achieved on various examples as we show below. However, it does not seem easy to build such a $d$ with differentiability properties for $x \neq y$ without involving complicated expressions which are difficult to manipulate. Our main example of $d$ will not be differentiable for $x \neq y$. However, if $d(x, y)$ is differentiable for $x \in \partial \Omega, y \in \Omega$ (as it will be the case for our main example), then (H5) holds as soon as we have

$$
\begin{aligned}
& H\left(x, t, s, \lambda \nabla_{x} d(x, y)\right)+c n(x) \geq H\left(x, t, s, \lambda \nabla_{x} d(x, y)\right)- \\
& \omega(\lambda d+d,|s|) \text { for all } x \in \partial \Omega, y \in \Omega, t \in[0, T], s \in R, c \geq \\
& 0, \lambda \geq \lambda_{0}
\end{aligned}
$$

and (H5) holds on $\Omega \times \Omega$.

Before giving some complementary uniqueness results (obtained with a stronger variant of (45)), we present our basic example of $d(x, y)$ and explain on a few examples how it is possible to check (H5) or (42). The choice for $d$ we propose is

$$
d(x, y)=|x-y|+C_{0}|\tilde{d}(x)-\tilde{d}(y)|
$$

where $C_{0}$ is a positive constant that we may choose as we want and $\tilde{d}(x)=$ $d(x) \zeta(x)$.

Here, $d(x)=\operatorname{dist}(x, \partial \Omega)$ while $\zeta$ is a cut-off function that will always satisfy at least

$$
\begin{aligned}
& \zeta \in C^{1}(\Omega), 0 \leq \zeta \leq 1, \zeta=1 \text { near } \partial \Omega,\{x \in \Omega / \zeta(x)>0\} \subset \\
& \left\{x \in \Omega / d(x)<\varepsilon_{0}\right\},
\end{aligned}
$$

where $\varepsilon_{0}$ is a positive constant that we may choose as small as we wish, and $\varepsilon_{0}$ is always small enough so that $d$ is differentiable on $\left\{x \in \Omega / d(x) \leq \varepsilon_{0}\right\}$.

Let us immediately give a result showing how to check (H5). 
Proposition V.1. Assume that $H$ satisfies (H2), (H3) and

There exists a positive constant $C_{1}$ such that for all $x \in \partial \Omega, p \in$ $R^{N}$, the function $\lambda \rightarrow H(x, t, s, p+\lambda n(x))$ is nondecreasing for $\lambda \geq C_{1}(1+|p|)$.

Then, $d$ given by (43) satisfies (H5).

Proof. First of all, we observe that $d(x, y)$ given by (43) is differentiable for $x, y \in \bar{\Omega} \times \bar{\Omega}$ such that $\tilde{d}(x) \neq \tilde{d}(y)$ or $x \neq y$ and $\tilde{d}(x)=\tilde{d}(y)=0$. In particular, if $x \in \partial \Omega, y \in \Omega$ then $d$ is continuously differentiable in a neighborhood (relative to $\bar{\Omega} \times \bar{\Omega})$ of $(x, y)$. Therefore, we only have to check (42) and to show that (H5) holds on $\Omega \times \Omega$ with some local modulus depending only on $c_{0}$ and $\zeta$.

For the latter claim, we approximate $d(x, y)$ by

$$
d_{\varepsilon}(x, y)=\left(\varepsilon^{2}+|x-y|^{2}\right)^{1 / 2}+C_{0}\left(\varepsilon^{2}+(\tilde{d}(x)-\tilde{d}(y))^{2}\right)^{1 / 2}
$$

where $\varepsilon>0$. Now $d_{\varepsilon} \in C^{1}(\bar{\Omega} \times \bar{\Omega})$ and

$$
\begin{aligned}
H\left(x, t, s, \lambda \nabla_{x} d_{\varepsilon}\right)-H\left(y, t, s,-\lambda \nabla_{y} d_{\varepsilon}\right) & \\
= & \left.H\left(x, t, s, \lambda \nabla_{x} d_{\varepsilon}\right)-H\left(y, t, s, \lambda \nabla_{x} d_{\varepsilon}\right)\right) \\
\quad & +\left(H\left(y, t, s, \lambda \nabla_{x} d_{\varepsilon}\right)-H\left(y, t, s,-\lambda \nabla_{y} d_{\varepsilon}\right)\right) .
\end{aligned}
$$

The first term is bounded from below by

$$
-\omega(|x-y|(1+\lambda+K \lambda)+|x-y|,|s|)
$$

for some $K$ depending only on $C_{0}, \zeta$. Here we use (H2). Next, choosing $\zeta$ in such a way that $\zeta \equiv 0$ on $\Gamma_{0}^{c}$, we see that (replacing of necessary $x$ by $y$ ) we may assume that $y \in \Gamma_{0}$ to bound the second term. In that case the second difference is bounded from below by

$$
-\mu\left(\lambda C_{0}|\nabla \tilde{d}(x)-\nabla \tilde{d}(y)|,|s|\right) \geq-\mu(\lambda k|x-y|,|s|)
$$

using (H3). Therefore (H5) holds with $z=\lambda d_{\varepsilon}$ for some $\omega$ independent of $\varepsilon$ and we conclude that (H5) holds on $\Omega \times \Omega$ letting $\varepsilon$ go to 0 .

We now check (42) and this is where we will determine $C_{0}$. Indeed we compute for $x \in \partial \Omega, y \in \Omega$

$$
\begin{aligned}
& H\left(x, t, s, \lambda \nabla_{x} d(x, y)+c n(x)\right)-H\left(x, t, s, \lambda \nabla_{x} d(x, y)\right) \\
& \quad=H\left(x, t, s, \lambda \frac{x-y}{|x-y|}+\left(\lambda C_{0}+c\right) n(x)\right)-H\left(x, t, s, \lambda \frac{x-y}{|x-y|}+\lambda C_{0} n(x)\right) .
\end{aligned}
$$

By (45), this difference is nonnegative for $\lambda \geq 1$ provided $\lambda C_{0} \geq C_{1}(1+\lambda)$ and this is the case if we take $C_{0}=2 C_{1}$.

Remark. It is quite obvious that the choice of $d$ given by (43) yields a particular emphasis on $|p|$ (see condition (45) for example) and it is easy to give other possible choices for $d$ leading to different conditions.

We now give a series of examples showing various situations in which (45) holds. 
Example V.1. Take $H(x, t, s, p)=F(x, t, s,|p|)$ where $F$ is nondecreasing with respect to $p$ for $|p| \geq R_{0} \geq 0$. Then, assuming that $|p+\lambda u|$ is nondecreasing for $\lambda \geq|p|$, we deduce easily that (45) holds.

Example V.2. Assume that $H(x, t, s, p)$ is given by

$$
H(x, t, p)=\sup _{\alpha \in A}\left[-b_{\alpha}(x, t) \cdot p-f_{\alpha}(x, t)\right]
$$

where $A$ is a given set and $b_{\alpha}(x, t), f_{\alpha}(x, t)$ are bounded uniformly in $\alpha \in A$. Then, (45) holds if we assume

$$
\exists \nu>0, \forall x \in \partial \Omega, \forall t \in[0, T], \exists \alpha \in A, \quad b_{\alpha}(x, t) \cdot n(x) \leq-\nu<0 .
$$

Indeed, we first observe that for $\lambda \geq 0$

$$
H(x, t, p+\lambda n) \geq \lambda \nu-c(1+|p|),
$$

for some $c \geq 0$. Therefore there exists a constant $C_{1} \geq 0$ such that $H(x, t, p+\lambda n)=\sup \left[-b_{\alpha}(x, t) \cdot p+\lambda n-f_{\alpha}(x, t), \alpha \in A, b_{\alpha}(x, t) \cdot n(x) \leq 0\right]$ if $(x, t) \in \partial \Omega \times[0, T], p \in R^{N}$ and $\lambda \geq C_{1}(1+|p|)$. It is then straightforward to check (45).

Example V.3. Assume that $H(x, t, s, p)$ is given by

$$
H(x, t, p)=\inf _{\alpha \in A}\left[-b_{\alpha}(x, t) \cdot p-f_{\alpha}(x, t)\right]
$$

where $A$ is a given set and $b_{\alpha}, f_{\alpha}$ are bounded uniformly in $\alpha \in A$. Then, (45) holds if we assume

$$
\forall x \in \partial \Omega, \forall t \in[0, T], \forall \alpha \in A, \quad b_{\alpha}(x, t) \cdot n(x) \leq 0 .
$$

Example V.4. Assume that $H(x, t, s, p)$ is given by

$$
H(x, t, p)=\inf _{\alpha \in A} \sup _{\beta \in B}\left[-b_{\alpha, \beta}(x, t) \cdot p-f_{\alpha, \beta}(x, t)\right]
$$

or by

$$
H(x, t, p)=\sup _{\beta \in B} \inf _{\alpha \in A}\left[-b_{\alpha, \beta}(x, t) \cdot p-f_{\alpha, \beta}(x, t)\right] .
$$

Then, (45) holds in the first case if we assume

$$
\begin{gathered}
\exists \nu>0, \forall x \in \partial \Omega, \forall t \in[0, T), \forall \alpha \in A, \exists \beta \in B, \\
b_{\alpha, \beta}(x, t) \cdot n(x) \leq-\nu<0
\end{gathered}
$$

and in the second case if we have

$$
\begin{gathered}
\exists \nu>0, \forall x \in \partial \Omega, \forall t \in[0, T), \exists \beta \in B, \forall \alpha \in A, \\
b_{\alpha, \beta}(x, t) \cdot n(x) \leq-\nu<0 .
\end{gathered}
$$


We now present some uniqueness result. We will use a stronger form of (H5); in fact we will need to complement (H5) with

There exists $d_{n}(x, y) \in C^{1}(\bar{\Omega} \times \bar{\Omega})$ such that for all $x, y \in$ $\bar{\Omega}, t \in[0 T], s \in R, \lambda \geq \lambda_{0}$

$$
\begin{aligned}
& \qquad \begin{aligned}
H\left(x, t, s, \lambda \nabla_{x} d_{n}(x, y)\right)-H\left(y, t, s,-\lambda \nabla_{y} d_{n}(x, y)\right) \\
\quad \geq-\omega\left(\lambda d_{n}+d_{n},|s|\right)
\end{aligned} \\
& \text { and } d_{n} \rightarrow d \text { uniformly on } \bar{\Omega} \times \bar{\Omega} \text {. }
\end{aligned}
$$

$d$ is differentiable with respect to $x$ if $x$ belongs to a neighborhood of $\partial \Omega$ and $\operatorname{dist}(x, \partial \Omega)<\operatorname{dist}(y, \partial \Omega)$.

$$
\begin{aligned}
& \text { If } y \in \Omega, x_{m} \in \Omega, x_{m} \rightarrow x_{0} \in \partial \Omega, \lambda_{m} \rightarrow+\infty \\
& \begin{array}{l}
H\left(x_{m}, t, s, \lambda \nabla_{x} d\left(x_{m}, y\right)+\lambda n\left(x_{m}\right)\right)-H\left(x_{m}, t, s, \lambda \nabla_{x} d\left(x_{m}, y\right)\right) \\
\geq-\omega(\lambda d+d,|s|) .
\end{array}
\end{aligned}
$$

In the typical case discussed above $d(x, y)=|x-y|+C_{0}|\tilde{d}(x)-\tilde{d}(y)|$, then we may choose

$$
d_{n}(x, y)=\left(\frac{1}{n}+|x-y|^{2}\right)^{1 / 2}+C_{0}\left(\frac{1}{n}+\left(d(\tilde{x})-d(\tilde{y})^{2}\right)\right)^{1 / 2}
$$

in order to check $(\mathrm{H} 6)$. And $(\mathrm{H} 6)-(\mathrm{H} 8)$ hold in all the examples discussed above. We may now state our uniqueness result (recall that we consider here only the case $\Omega$ bounded).

Theorem V.1. Assume (H1), (H5)-(H8).

(1) (Stationary problem) Let $u, v \in C(\bar{\Omega})$ be, respectively, a viscosity subsolution of (11) in $\Omega$, and a viscosity supersolution of (13) for some $f \in C(\overline{\mathbf{\Omega}})$. Then (14) holds.

(2) (Cauchy problem) Let $u, v \in C(\bar{\Omega} \times[0, T])$ be respectively a viscosity subsolution of (12), and a viscosity supersolution of (15) for some $f \in$ $C(\bar{\Omega} \times[0, T])$. Then (16) holds.

Proof. We will detail only the case of the stationary problem, and to simplify the presentation we will assume that $H$ depends only on $(x, p), \omega$ does not depend on $|s|$. The idea of the proof is to build a supersolution $z(x, y)$ using $d(x, y)$ and then to prove that the function

$$
\max _{x \in \bar{\Omega}}\{u(x)-z(x, y)\}=\tilde{u}(y)
$$

is still a viscosity subsolution of (11). Since $\tilde{u}$ will be finally Lipschitz on $\bar{\Omega}$, we conclude applying Theorems III.1 and 2.

To build $z$, we observe, in view of the constructions made in M. G. Crandall and P.-L. Lions [9, 10], M. G. Crandall, H. Ishii and P.-L. Lions [7], that we 
can find $C \geq 0, \delta_{0}>0, \gamma \in(0,1)$ such that $z_{n}(x, y)=C\left(\delta^{2}+d_{n}(x, y)^{2}\right)^{\gamma / 2}$ is a $C^{1}$ supersolution of

$$
z_{n}+H\left(x, \nabla_{x} z_{n}\right)-H\left(y,-\nabla_{y} z_{n}\right) \geq \omega\left(z_{n}\right) \quad \text { in } \Omega \times \Omega
$$

for $\delta<\delta_{0}$. And we set $z(x, y)=C\left(\delta^{2}+d(x, y)^{\varepsilon}\right)^{\gamma / 2}$; in fact $z$ depends upon $\delta$ and we will recall this dependence only at the end of the proof.

We next define $\tilde{u}$ by (54) and $\tilde{u}_{n}$ similarly replacing $z$ by $z_{n}$. We want to prove that $\tilde{u}$ is a viscosity subsolution of (11). We first consider the open set $\Omega_{0}=\{y \in \Omega / u(x)-z(x, y)$ is maximum on $\bar{\Omega}$ only at points $x$ in $\Omega\}$. Therefore for $n$ large enough

$\left[\tilde{u}_{n}(y)=u(x)-z_{n}(x, y), x \in \bar{\Omega}\right]$ implies $x \in \Omega$. Let $\phi \in C^{1}(\bar{\Omega})$ and let $y_{0}$ be a point in $\Omega_{0}$ such that $\tilde{u}_{n}-\phi$ has a local maximum at $y_{0}$. Next, let $x_{0} \in \bar{\Omega}$ be a maximum over $\bar{\Omega}$ of $u-z_{n}\left(x, y_{0}\right)$. Obviously, $\nabla \phi\left(y_{0}\right)=-\nabla_{y} z_{n}\left(x_{0}, y_{0}\right)$, $\nabla_{x} z_{n}\left(x_{0}, y_{0}\right) \in D^{+} u\left(x_{0}\right)$. Thus we find

$$
\begin{gathered}
u\left(x_{0}\right)+H\left(x_{0}, \nabla_{x} z_{n}\left(x_{0}, y_{0}\right)\right) \leq 0 \\
z_{n}\left(x_{0}, y_{0}\right)+H\left(x_{0}, \nabla_{x} z_{n}\left(x_{0}, y_{0}\right)\right)-H\left(y_{0}, \nabla \phi\left(y_{0}\right)\right) \geq 0
\end{gathered}
$$

and subtracting we obtain

$$
\tilde{u}_{n}\left(y_{0}\right)+H\left(y_{0}, \nabla \phi\left(y_{0}\right)\right) \leq 0 .
$$

We then easily deduce from these considerations that $\tilde{u}$ is a viscosity subsolution of (11) in $\Omega_{0}$. Next, let $K$ be a compact set in $\Omega$ and let $y_{0} \in K-\Omega_{0}$; we just have to prove that $\tilde{u}$ is a viscosity subsolution of $(11)$ in an open neighborhood of $y_{0}$. We choose a ball around $y_{0}$ small enough so that it is contained in a compact set of $\Omega$. Let $\phi \in C^{1}(\bar{\Omega})$ be such that $\tilde{u}-\phi$ has a local maximum at a point $y_{1}$ in that ball. Next, if one maximum point of $u-z\left(\cdot, y_{0}\right)$ lies in $\Omega$ we argue as before, while if all maximum points of $u-z\left(\cdot, y_{0}\right)$ are on $\partial \Omega$, we consider

$$
\tilde{u}_{\varepsilon}(y)=\max _{x \in \bar{\Omega}} u(x)-z(x, y)-\frac{\varepsilon}{d(x)}
$$

with $\varepsilon>0, d(x)=\operatorname{dist}(x, \partial \Omega)$. Obviously the maximum, for $y=y_{0}$ is achieved at some point $x_{\varepsilon} \in \Omega$ and we may assume, taking subsequences if necessary, that $x_{\varepsilon}$ converges to $x_{0} \in \partial \Omega$ as $\varepsilon$ goes to 0 where $x_{0}$ is a maximum point of $u(\cdot)-z\left(\cdot, y_{0}\right)$. We may now use the various properties of $u, z, H$ to write

$$
\begin{gathered}
u\left(x_{\varepsilon}\right)+H\left(x_{\varepsilon}, \nabla_{x} z\left(x_{\varepsilon}, y_{0}\right)+\frac{\varepsilon}{d^{2}\left(x_{\varepsilon}\right)} n\left(x_{\varepsilon}\right)\right) \leq 0, \\
z\left(x_{\varepsilon}, y_{0}\right)+H\left(x_{\varepsilon}, \nabla_{x} z\left(x_{\varepsilon}, y_{0}\right)\right)-H\left(y_{0},-\nabla_{y} z\left(x_{\varepsilon}, y_{0}\right)\right) \geq \omega(z) \geq 0
\end{gathered}
$$

and we conclude using (H8) (indeed, if $\varepsilon / d^{2}\left(x_{\varepsilon}\right)$ remains bounded, we use (H5)). 
Hence $\tilde{u}$ is Lipschitz on $\Omega$ and is a viscosity subsolution of (11). We may now use Theorem III. 1 to deduce

$$
\max _{\bar{\Omega}}(\tilde{u}-v)^{+} \leq \max _{\bar{\Omega}} f^{+} .
$$

To conclude we observe that $\tilde{u} \geq u-C \delta^{\gamma}$ and we conclude sending $\delta$ to 0 .

\section{General EXISTENCE RESUltS}

We will prove existence in two different situations: the first one is when (H5) holds. To simplify the presentation, we will still assume that $\Omega$ is bounded. Our main existence results are then:

Theorem VI.1 (The stationary problem). Assume (H1) and (H5). Then there exists $u \in C(\bar{\Omega})$ viscosity solution of (11) and viscosity supersolution on $\bar{\Omega}$ such that $v \geq$ on $\bar{\Omega}$ for any $v \in C(\bar{\Omega})$ viscosity supersolution of (11) on $\bar{\Omega}$.

Theorem VI.2 (The Cauchy problem). Assume (H1) and (H5). Let $u_{0} \in C(\bar{\Omega})$. Then there exists $u \in C(\bar{\Omega} \times[0, T])$ viscosity solution of (12) and viscosity supersolution on $\bar{\Omega} \times] 0, T\left[\right.$ satisfying $u(\cdot, 0)=u_{0}(\cdot)$ on $\bar{\Omega}$ and such that for any $u \in C(\bar{\Omega} \times[0, T])$ viscosity supersolution on $\bar{\Omega} \times] 0, T\left[\right.$ with $v(\cdot, 0) \geq u_{0}(\cdot)$ on $\bar{\Omega}$ then $v \geq u$ on $\bar{\Omega} \times[0, T]$.

Remarks. (i) We do not know if the solutions built above are unique. Of course, strengthening (H5) to $(\mathrm{H} 5)-(\mathrm{H} 8)$, we obtain uniqueness in view of $\S \mathrm{V}$.

(ii) If the modulus $\omega$ can be taken of the form $C_{1}(|x-y|)+C_{2}(|s|)$, then the method of proof below yields a solution which is Hölder continuous with an exponent $\theta=\operatorname{Min}\left(1 / C_{1}(M), 1\right)$ if $\left.C_{1} \neq 1, \theta \in\right] 0,1\left[\right.$ if $C_{1}=1$ in the case of (11) where $M=\|H(x, 0,0)\|_{\infty}$, while in the case of (12) the solution built is Lipschitz on $\bar{\Omega} \times[0, T]$ if $u_{0}$ is Lipschitz on $\bar{\Omega}$. Notice also that in this case the solution built is unique in the class mentioned at the end of $\S I V$.

Proof of Theorem VI.1. Even if we could use the same line of arguments in both theorems, we prefer to give a shorter proof in the case of (11). If $M$ is the constant given in the remark above we consider, following G. Barles [1], the new Hamiltonian $\tilde{H}(x, s, p)=T_{M} H\left(x, T_{m} s, p\right)$ where

$$
T_{M} \lambda=\operatorname{Max}(\operatorname{Min}(\lambda, M),-M)
$$

for all $\lambda \in R$. Assumptions (H1) and (H5) (with $\omega$ now independent of $s$ ) are still satisfied and simple arguments show that we only have to solve our problem for this new Hamiltonian.

To do so, we consider Hamiltonians $H_{\varepsilon}(x, s, p)=H(x, s, p)+\varepsilon|p|$. By Theorem IV.1, there exists a unique $u_{\varepsilon} \in W^{1, \infty}(\Omega)$ viscosity solution in $\Omega$ and viscosity supersolution in $\bar{\Omega}$ of $u_{\varepsilon}+H_{\varepsilon}\left(x, u_{\varepsilon}, \nabla u_{\varepsilon}\right)=0$ in $\Omega$ and, obviously, $\left|u_{\varepsilon}\right| \leq M$ on $\Omega$. Because of (H5), as in [10,7], we can find $C>0, \gamma \in(0,1)$, $\delta_{0}>0$ such that for $0<\delta \leq \delta_{0}$

$$
w_{\delta}(x, y)=C\left(\delta^{2}+d(x, y)^{2}\right)^{y / 2}
$$


is a viscosity supersolution on $\bar{\Omega} \times \Omega$ of

$$
w_{\delta}+\inf _{t}\left\{H\left(x, t, \nabla_{x} w_{\delta}\right)-H\left(y, t,-\nabla_{y} w_{\delta}\right)\right\} \geq 0 .
$$

Now, using the results and methods of $\S I I I$, we deduce

$$
\left|u_{\varepsilon}(x)-u_{\varepsilon}(y)\right| \leq w_{\delta}(x, y)+\varepsilon C_{\delta} \text { on } \bar{\Omega} \times \bar{\Omega}
$$

for some positive constant $C_{\delta}$ depending only on $\delta$. Therefore, $u_{\varepsilon}$ has an approximated modulus of continuity

$$
\left|u_{\varepsilon}(x)-u_{\varepsilon}(y)\right| \leq \omega_{\varepsilon}(|x-y|) \quad \text { on } \bar{\Omega} \times \bar{\Omega}
$$

where $\omega_{\varepsilon}$ is continuous, nonnegative, nondecreasing, subadditive and $\omega_{\varepsilon}(t)$ converges as $\varepsilon$ goes to 0 to a modulus $\omega(t)$ (i.e. $\omega_{\varepsilon}(t)$ converges to $\omega(t)$ and $\omega(0)=0)$. Indeed, (55) implies (56) with the choice

$$
\omega_{\varepsilon}(t)=\inf _{0<\delta<\delta_{0}}\left\{C\left(\delta^{2}+k t^{2}\right)^{i / 2}+\varepsilon C_{\delta}\right\}
$$

where $K$ is a Lipschitz constant for $d(x, y) \quad(d(x, y) \leq K|x-y|$ for all $x, y)$.

Next, we claim that since $u_{\varepsilon}$ is uniformly bounded and since $u_{\varepsilon}$ satisfies (56), then $u_{\varepsilon}$ is relatively compact in $C(\bar{\Omega})$ as $\varepsilon$ goes to 0 . This is a simple variation of Ascoli's theorem whose verification we leave to the reader as an exercise. Noticing that $u_{\varepsilon}$ is nonincreasing with respect to $\varepsilon$ we finally deduce that $u_{\varepsilon}$ converges in $C(\bar{\Omega})$ to some $u$. There just remains to show that if $v$ is any viscosity supersolution of (11) on $\bar{\Omega}$ then $v \geq u$ in $\bar{\Omega}$. This is very easy since $v$ is clearly a viscosity supersolution of (11) on $\bar{\Omega}$ where $H$ is replaced by $H_{\varepsilon}$ for all $\varepsilon>0$. Now, since $u_{\varepsilon}$ is Lipschitz, we deduce from $\S$ III that $v \geq u_{\varepsilon}$ and we conclude passing to the limit.

Proof of Theorem VI.2. First, considering $H\left(x, t, T_{M} s, p\right)$ instead of $H$ where

$$
M=\left\|u_{0}\right\|_{\infty}+T \sup _{t \geq 0}\|H(x, t, 0,0)\|_{\infty},
$$

we see that we may assume that only bounded values of $s$ matter. Next, we introduce a sequence of Hamiltonians $\tilde{H}_{\varepsilon}(x, t, s, p)$ nondecreasing in $s$, smooth in $t$, continuous in all variables satisfying: $\tilde{H}_{\varepsilon}(x, t, s, p) \downarrow H(x, t, s, p)$ as $\varepsilon \downarrow 0$ uniformly for $x \in \bar{\Omega}, t \in[0, T], s \in R, p$ bounded and thus

$$
\left|\tilde{H}_{\varepsilon}(x, t, s, p)-H(x, t, s, p)\right| \leq \mu(\varepsilon, 1+|p|)
$$

for some local modulus $\mu$. We also consider $u_{0}^{\varepsilon} \in W^{1, \infty}(\Omega)$ such that $u_{0}^{\varepsilon} \uparrow u_{0}$ in $\bar{\Omega}$ as $\varepsilon \downarrow 0$ uniformly on $\bar{\Omega}$. In particular, there exists a nonnegative, continuous, nondecreasing function $\Phi$ on $[0, \infty[$ such that

$$
\left|\frac{\partial^{\alpha} \tilde{H}_{\varepsilon}}{\partial t^{\alpha}}(x, t, s, p)\right| \leq \Phi(1+|p|) \quad \text { for } \alpha=0,1 \text {. }
$$

And we finally consider a nonnegative, continuous, nondecreasing function $\Psi$ on $[0, \infty[$ such that

$$
\Psi / \Phi \rightarrow+\infty \text { as } t \rightarrow+\infty
$$


We set $H_{\varepsilon}(x, t, s, p)=\tilde{H}_{\varepsilon}(x, t, s, p)+\varepsilon \Psi(|p|)$ and we claim there exists a unique Lipschitz function $u_{\varepsilon}$ on $\bar{\Omega} \times[0, T]$ viscosity solution in $\Omega \times(0, T)$ and viscosity supersolution in $\bar{\Omega} \times(0, T)$ of

$$
\begin{aligned}
& \partial u_{\varepsilon} / \partial t+H_{\varepsilon}\left(x, t, u_{\varepsilon}, \nabla u_{\varepsilon}\right)=0 \quad \text { in } \Omega \times(0, T), \\
& \left.u_{\varepsilon}\right|_{t=0}=u_{0}^{\varepsilon} \text { in } \Omega .
\end{aligned}
$$

This claim is verified by the same proof as in Theorem V.2 using the remarks of G. Barles and P.-L. Lions [3] (the idea being heuristically

$$
\left\|\frac{\partial u_{\varepsilon}}{\partial t}\right\|_{\infty} \leq T \Phi\left(1+\left\|\nabla u_{\varepsilon}\right\|_{\infty}\right)+\Phi\left(1+\nabla u_{\varepsilon}^{0} \|_{\infty}\right)
$$

while from the equation one "deduces"

$$
\varepsilon \Psi\left(\left\|\nabla u_{\varepsilon}\right\|_{\infty}\right) \leq(1+T) \Phi\left(1+\left\|\nabla u_{\varepsilon}\right\|_{\infty}\right)+\Phi\left(1+\left\|\nabla u_{\varepsilon}^{0}\right\|_{\infty}\right)
$$

and thus a formal Lipschitz bound is derived using (57)).

Obviously, $u_{\varepsilon}$ is uniformly bounded (for $\varepsilon$ say $\leq 1$ ) and we just need to obtain an approximate modulus of continuity of $u_{\varepsilon}$ in $x$ uniform in $t:$ indeed, this yields as in M. G. Crandall and P.-L. Lions [10] an approximate modulus of continuity in $(x, t)$ and then one concludes as in the proof of Theorem VI.1. To obtain this approximate modulus we argue as in Theorem VI.1 and we find instead of (55) for $0<\delta \leq S_{0}$

$$
\left|u_{\varepsilon}(x, t)-u_{\varepsilon}(y, t)\right| \leq C_{1} e^{c_{2} t}\left(\delta^{2}+d(x, y)^{2}\right)^{y / 2}+\mu\left(\varepsilon, C_{\delta}\right)+\varepsilon \Psi\left(C_{\delta}\right)
$$

for some constants $C_{1}, C_{2}>0, \gamma \in(0,1), \delta_{0}>0$, independent of $\varepsilon$ and $\delta$ and for some constant $C_{\delta}$ depending only on $\delta$ and we conclude.

Our final existence result requires very strong assumptions near $\partial \Omega$ but we believe that it is worth mentioning because it is not contained in the preceding results stricto sensu and the method of proof is very natural. We will need to assume that there exists a tubular neighborhood $\tilde{\Gamma}$ of $\partial \boldsymbol{\Omega}$ (relative to $\bar{\Omega}$ ) and a local modulus $m$ such that

$$
\begin{aligned}
& |H(x, t, s, p)-H(y, t, s, p)| \leq m(|x-y|,|s|) \\
& \text { for all } x, y \in \tilde{\Gamma}, t \in[0, T], s \in R, p \in R^{N},
\end{aligned}
$$

$$
\begin{aligned}
& H(x, t, s, p+\lambda n(x)) \rightarrow+\infty \text { as } \lambda /(1+|p|) \rightarrow \infty \text { uniformly for } \\
& x \in \tilde{\Gamma}, t \in[0, T],|s| \leq R,
\end{aligned}
$$

where $n$ denotes as usual $n(x)=-\nabla d(x)$ near $\partial \Omega$. Let us emphasize that in the following results we do not assume anymore that $\Omega$ is bounded.

Theorem VI.3. Assume (H1)-(H3) and (59)-(60). Assume finally that $H \in$ $B U C\left(\bar{\Omega} \times[0, T] \times[-R,+R] \times \bar{B}_{R}\right) \quad(\forall R<\infty)$.

1. (Stationary problem) There exists a unique viscosity solution in $B U C(\Omega)$ of (11) which is a viscosity supersolution of (11) on $\bar{\Omega}$. 
2. (Cauchy problem) Let $u_{0} \in B U C(\bar{\Omega})$ and assume that (49) holds. There exists a unique viscosity solution in $B U C(\bar{\Omega} \times[0, T])$ of (12) which is a viscosity supersolution of (12) on $\bar{\Omega} \times(0, T)$.

Sketch of a proof. We only sketch the proof of the above assertion in the case of the Cauchy problem. We claim that, using the methods of M. G. Crandall and P.-L. Lions [9], it is enough to obtain a priori estimate on the modulus of continuity of solutions near $\partial \Omega$. We first claim it is enough to work with $H$ satisfying (H2), (59) with moduli independent of $|s|$ and of the form $m(t)=C t$ for some constant $C \geq 0$. The independence of $|s|$ is achieved by a standard truncation as before and a "Lipschitz modulus" $m$ in (59) is obtained as in $\S \mathrm{V}$. Then the reduction to "Lipschitz moduli" is obtained by standard mollifications $H * p_{\varepsilon}$ and by choosing a cut-off function $\phi \in \mathscr{D}(\Omega), 0 \leq \phi \leq 1$ in $\Omega, \Phi \equiv 0$ on $\tilde{\Gamma}$. Indeed one considers

$$
H_{\varepsilon}=\phi(x) H(x, t, s, p)+(1-\phi(x)) \iint H(y, t, s, q) p_{\varepsilon}(x-y) p_{\varepsilon}(p-q) d y d q
$$

for some mollifier $p_{\varepsilon}=p(\cdot / \varepsilon) / \varepsilon^{N}$ with $p \in \mathscr{D}\left(R^{N}\right), p \geq 0, \int_{R^{*}} p d x=1$. Then (H1) still holds for $H_{\varepsilon}$; (H2), (H3), (59), (60) hold uniformly in $\varepsilon \in$ $(0,1)$ and $(\mathrm{H} 3),(59)$ hold with "Lipschitz moduli". Obviously, $H_{\varepsilon}$ converges uniformly to $H$. Hence, we just have to prove the existence of a solution $u_{\varepsilon}$ for equation (11) where $H$ is replaced by $H_{\varepsilon}$. Finally, by density, it is enough to consider the case when $u_{0} \in W^{1, \infty}(\Omega)$.

Now, if the moduli in (H3) and (H2) are Lipschitz we may use to obtain a priori estimates the simple method of translations. We are in fact going to prove that in this case the solutions are Lipschitz near the boundary. Indeed let $\tau$ be a vector field smooth on $\bar{\Omega}$, say $C^{1}$, such that $\tau$ vanishes on $\bar{\Omega}-\tilde{\Gamma}$ and $\tau \cdot n(x)=0$ in $\tilde{\Gamma}$. Denote by $u_{h}(x, t)=u(X(h, x), t)$ where $u$ is the Lipschitz viscosity solution of (11), supersolution on $\bar{\Omega}$ and where $X(h, x)$ is the solution at time $t$ of the O.D.E. $\dot{X}_{t}=\tau\left(X_{t}\right), X_{0}=x$. Clearly $u_{h}$ is a viscosity solution in $\Omega \times(0, T)$ and supersolution in $\bar{\Omega} \times(0, T)$ of

$$
\frac{\partial u_{h}}{\partial t}+H\left(X(h, x), t, u_{h}, T_{h}(x) \cdot \nabla u_{h}(x)\right)=0
$$

where $T_{h}$ satisfies $\left\|T_{h}-I\right\|_{\infty} \leq c h,\left|T_{h}\left(x_{1}\right)-T_{h}\left(x_{2}\right)\right| \leq C h\left(x_{1}-x_{2}\right)$ for $h \in[0,1]$. In particular we find (in viscosity sense)

$$
\frac{\partial u_{h}}{\partial t}+H\left(x, t, u_{h}, \nabla u_{h}\right)=G_{h}\left(x, t, s, \nabla u_{h}(x)\right)
$$

where $\left|G_{h}\right| \leq C h(1+|p|)$ for all $x \in \bar{\Omega}, p \in R^{N}, h \in[0, T], t \in[0, T]$, $s \in R$. Therefore, by the comparison results of $\S I I I$, we deduce

$$
\sup _{x \in \bar{\Omega}}|u(x, t)-u(X(h, x), t)| \leq C h\left(1+\int_{0}^{t}\|\nabla u(s)\|_{\infty} d s\right) \text { for all } t \in[0, T] .
$$


By a similar method, one obtains for $h$ small enough

$$
\sup _{x \in \bar{\Omega}}\{u(x-h n(x), t)-u(x)\}^{+} \leq c h\left(1+\int_{0}^{t}\|\nabla u(x)\|_{\infty} d s\right) .
$$

Remarking that as in $\S \mathrm{V}$ we may obtain the estimate

$$
u(x, t+h)-u(x, t) \geq-C h \text { for } x \in \bar{\Omega}, 0 \leq t \leq t+h \leq T,
$$

we deduce from the equation, combining (61)-(63) and (60),

$$
\|\nabla u(t)\|_{L^{\infty}(\tilde{\Gamma})} \leq C+c \int_{0}^{t}\|\nabla u(s)\|_{L^{\infty}(\Omega)} d s
$$

for all $t \in[0, T]$.

To conclude, we use the fact that, by Barles method [1], we can assume without loss of generality that $H$ satisfies

$$
|H(x, t, s, p)-H(y, t, s, p)| \leq C|x-y|(1+|p|)
$$

for all $x, y \in \bar{\Omega}, t \in[0, T), s \in R, p \in R^{N}$ and for some constant $C \geq 0$. And by standard comparison argument, we deduce from the equation and (64)

$$
\|\nabla u(t)\|_{L^{\infty}(\Omega)} \leq C+c \int_{0}^{t}\|\nabla u(s)\|_{L^{\infty}(\Omega)} d s
$$

and we conclude.

Remarks. (i) If $\Omega$ is a half-space, then by the same method one may relax (60) replacing $\lambda /(1+|p|)$ by $\lambda$, provided $p$ is bounded.

(ii) To simplify the presentation, we worked with $B U C$ solutions. As in [9, 10] we could work with $U C$ solutions or even unbounded solutions.

(iii) If $\Omega$ is bounded, (59) and (61) imply that $H(x, t, s, p) \rightarrow+\infty$ as $|p| \rightarrow \infty$ uniformly for $x \in \tilde{\Gamma}, t \in[0, T], s$ bounded. Indeed, if $p$ is arbitrary in $R^{N}$ then $p=n(y)|p|$ for some $y \in \partial \Omega$ and if $x \in \tilde{\Gamma}$

$$
H(x, t, s, p) \geq H(y, t, s,|p| n(y))-C
$$

in view of (59) and we conclude easily.

\section{APPROXIMATIONS}

In this section, we want to deal with three types of approximations of viscosity solutions which are supersolutions on the closure of the domain. The first one was already used in $\S I V$ : the so-called penalty approach. The second one is simply to build the upper envelope of viscosity subsolutions and the third one is an approximation from above by viscosity solutions of approximated problems in $\Omega$ which blow up the boundary (see J. M. Lasry and P.-L. Lions [22], M. G. Crandall and P.-L. Lions [11] for other examples of uses of such solutions in different problems). We will explain the various approaches and results only on the example of the stationary problem (11) but everything adapts to the case of the Cauchy problem without any changes. To simplify the presentation, we 
only consider the case of a bounded domain $\Omega$. We assume one extends $H$ to $R^{N} \times R \times R^{N}$ in such a way that for example $H \in B U C\left(R^{N} \times[-R,+R] \times \bar{B}_{R}\right)$ for all $R<\infty$. Let $p \in B U C\left(R^{N}\right)$ satisfy

$$
\forall \varepsilon>0, \exists \delta>0, p(x) \geq \delta \text { if } \operatorname{dist}(x, \bar{\Omega}) \geq \varepsilon, p \equiv 0 \text { on } \bar{\Omega} .
$$

We know (see $[9,10])$ that if we assume for instance

$$
|H(x, s, p)-H(y, s, p)| \leq \omega(|x-y|(1+|p|),|s|)
$$

for some local modulus $\omega$, then there exists a unique viscosity solution $u_{\varepsilon} \in$ $B U C\left(R^{N}\right)$ of (37).

We wish to prove that $u_{\varepsilon}$ (which is nondecreasing with respect to $\varepsilon>0$ ) converges to a viscosity solution of (11) in $\bar{\Omega}$ which is a viscosity supersolution of (11) on $\bar{\omega}$. To this end, we introduce $\Omega^{\delta}=\left\{x \in R^{N} / \operatorname{dist}(x, \bar{\Omega}) \leq \delta\right\}$ and we assume that for $\delta>0$ small

$$
\begin{aligned}
& \exists u^{\delta} \in C\left(\overline{\Omega^{\delta}}\right) \text { viscosity solution of }(11) \text { on } \overline{\Omega^{\delta}} \text {, viscosity super- } \\
& \text { solution of }(11) \text { on } \overline{\Omega^{\delta}} \text { and }\left|u^{\delta}(x)-u^{\delta}(y)\right| \leq \mu(|x-y|) \text { for } \\
& x, y \in \overline{\Omega^{\delta}},
\end{aligned}
$$

where $\mu$ is a modulus independent of $\delta>0$ small enough. In fact, this assumption is satisfied in most of the existence results above if we strengthen a bit the assumptions we used. Furthermore, we see that if we may apply uniqueness results, then $u^{\delta} \uparrow$ as $\delta \downarrow$ and then (68) implies the following assumption:

$$
\begin{aligned}
& u^{\delta} \text { converges uniformly to some function } u \text { on } \bar{\Omega} \text { as } \delta \text { goes } \\
& \text { to } 0, u \text { is the maximum viscosity subsolution of }(11) \text { in } C(\bar{\Omega}) \text {. }
\end{aligned}
$$

We then have

Theorem VII.1. Assume (H1), (66)-(69). Then, as $\varepsilon$ goes to $0_{+}, u_{\varepsilon}$ converges uniformly on $\bar{\Omega}$ to $u$ which is a viscosity solution in $\Omega$ and viscosity supersolution in $\bar{\Omega}$ of (11).

Proof. Of course, (68), (69) imply the properties of $u$ stated above but they may also be deduced from the proof of the convergence of $u_{\varepsilon}$ we now present. For $\delta>0$, fixed there exists $\alpha>0$ such that $p(x) \leq \alpha$ if $x \in \Omega^{\delta / 2}$. Observe also that $u_{\varepsilon} \geq-C_{0}=-\|H(x, 0,0)\|_{L^{\infty}\left(R^{v}\right)}$. We are first going to show that $u_{\varepsilon} \uparrow+\infty$ as $\varepsilon \downarrow 0_{+}$uniformly on $\partial \Omega^{\delta}$. Let $\Phi \in C_{b}^{1}\left(R^{N}\right)$ be such that $0 \leq$ $\Phi \leq 1, \Phi \equiv 0$ on $\Omega^{\delta / 2}, \Phi \equiv 1$ on $R^{N}-\Omega^{\delta}$ and let $C_{\delta}=\|\nabla \Phi\|_{\infty}$. We wish to build a subsolution of (37) of the form $-C_{0}+k \Phi$ where $k=k_{\varepsilon, \delta} \uparrow \infty$ as $\varepsilon \downarrow 0_{+}$, for any $\delta>0$. Indeed we have

$$
-C_{0}+k \Phi+H\left(x,-C_{0}+k \Phi, k \nabla \phi\right)=-C_{0}+H\left(x,-C_{0}, 0\right) \leq 0 \text { in } \Omega^{\delta / 2},
$$

while on $R^{N}-\Omega^{\delta / 2}$ we have

$$
\begin{aligned}
& -C_{0}+k \Phi+H\left(x,-C_{0}+k \Phi, k \nabla \Phi\right) \\
& \quad \leq-C_{0}+k+\sup \left\{H(x, k, p) / x \in R^{N},|p| \leq C_{\delta} k\right\}
\end{aligned}
$$


and this is less than $\alpha / \varepsilon$ if $k \leq k_{\varepsilon, \delta}$ with $k_{\varepsilon, \delta} \uparrow+\infty$ as $\varepsilon \downarrow 0_{+}$. In particular, comparison results yield

$$
u_{\varepsilon} \geq-C_{0}+k_{\varepsilon, \delta} \quad \text { on } \partial \Omega_{\delta}
$$

and our claim is proved.

Next, $u^{\delta}$ is a viscosity subsolution of (11) on $\Omega^{\delta}$ while $u_{\varepsilon}$ is a viscosity supersolution of (11) on $\Omega^{\delta}$. Furthermore, for any $\delta>0, u_{\varepsilon} \geq u^{\delta}$ on $\partial \Omega^{\delta}$ for $\varepsilon$ small enough, thus $u_{\varepsilon} \geq u^{\delta}$ on $\overline{\Omega^{\delta}}$ for $\varepsilon \leq \varepsilon_{0}(\delta)$. We then conclude easily since $u^{\delta}$ converges to $u$ by (59) and since $u_{\varepsilon} \leq u$, again by (59).

Our final result on this penalty approximation is an estimate on the rate of convergence of this method: the result which follows is only one example of the type of results which can be obtained by this method. We will assume in addition to (68), (69) that

$$
\left|u^{\delta}-u\right| \leq C \delta \text { on } \bar{\Omega}
$$

for $\delta>0$ small enough and we will consider the case of a Hamiltonian $H(x, p)$ satisfying

$$
H(x, p) \leq C(1+|p|) \quad \text { on } R^{N} \times R^{N} .
$$

Assumption (70) may seem to be difficult to check: actually, it is not. Assume for instance that $H$ is locally Lipschitz in $x$ and $p$ in a neighborhood of $\partial \Omega$ and that $u$ is Lipschitz near $\partial \Omega$. Then we claim (70) holds. Indeed, applying the comparison results we see that $u^{\delta} \leq u$. On the other hand, it is not difficult to build operators $T_{\delta} \in C^{2}$ (for $\delta$ small enough) so that $T_{\delta}(x)=x$ off a neighborhood of $\partial \Omega, T_{\delta}$ maps $\bar{\Omega}$ onto $\overline{\Omega^{\delta}}$ and $\partial \Omega$ onto $\partial \Omega^{\delta}, T_{\delta}$ is a diffeomorphism from $\bar{\Omega}$ to $\overline{\Omega^{\delta}}$ and

$$
\sup _{x \in \bar{\Omega}}\left\{\left|T_{\delta}(x)-x\right|+\left|\nabla T_{\delta}(x)-I\right|\right\} \leq C \delta .
$$

Then considering $\tilde{u}_{\delta}(x)=u\left(T_{\delta}^{-1}(x)\right)$ on $\bar{\Omega}^{\delta}$, we check easily that $\tilde{u}_{\delta}$ is a Lipschitz viscosity subsolution on $\Omega^{\delta}$ of

$$
\tilde{u}_{\delta}+H\left(x, \nabla \tilde{u}_{\delta}\right) \leq C \delta \quad \text { in } \Omega^{\delta}
$$

and thus by the comparison results of $\S$ III we deduce

$$
\tilde{u}_{\delta} \leq u_{\delta}+C \delta \quad \text { on } \overline{\Omega^{\delta}}
$$

and (70) follows easily.

We may now state

Proposition VII.1. Under the assumptions of Theorem VII.1 and if in addition (70) holds and $H=H(x, p)$ satisfies (71) then

$$
u \geq u_{\varepsilon} \geq u-C \sqrt{\varepsilon} \text { in } \bar{\Omega}
$$

for some constant $C \geq 0$. 
Proof. We just observe that because of (71) we can take in the proof of Theorem VII. $1 k_{\varepsilon, \delta}=C\left(1+\delta^{2} / \varepsilon\right)$. And since $u^{\delta} \leq C$ on $\partial \Omega^{\delta}$, we may in fact choose $\delta=c \sqrt{\varepsilon}$ for some large constant $C \geq 0$ to get

$$
u^{\delta} \leq u_{\varepsilon} \quad \text { on } \overline{\Omega^{\delta}}
$$

In particular this combined with (70) yields

$$
u \geq u_{\varepsilon} \geq u^{\delta} \geq u-C \delta=u-C \sqrt{\varepsilon} \quad \text { on } \bar{\Omega}
$$

and (72) is proved.

The next result is a variant of Theorem IV.1. Its proof relies on the construction of the upper envelope of all viscosity subsolutions of (11) (see H. Ishii [16] for similar ideas in related problems and R. Gonzalez and E. Rofman [15] for numerical schemes which build the maximum subsolution of equations like (11)).

Theorem VII.2. Assume (H1) and either (34) or (35) and (H2). Then there exists a maximum viscosity subsolution $u \in C(\bar{\Omega})$ of (11) which is a viscosity supersolution on $\bar{\Omega}$.

Proof. Let us recall that the maximum of a finite number of viscosity subsolutions is a viscosity subsolution (see [6]) and that (34) (or (35) and H2)) imply that if $v \in C(\bar{\Omega})$ is a subsolution of (11) then there exists $\tilde{v} \in C(\bar{\Omega})$ which is a viscosity solution of (11) with $\tilde{v}=v$ on $\partial \Omega$ (see [23,1]). Let us consider now the maximization problem

$$
\max \left\{\int_{\Omega} v d x \mid v \in C(\bar{\Omega}), v \text { viscosity subsolution of }(11)\right\} .
$$

In view of the above remarks there exists a maximizing sequence $\left(v_{n}\right)_{n} \epsilon$ $C(\bar{\Omega})$ such that $v_{n}$ is a viscosity solution of $(11)$ and $\left(v_{n}\right)$ is nondecreasing with respect to $n$. Suppose temporarily that $v_{n}$ converges in $C(\bar{\Omega})$ to some $u$. Then, $u$ is a viscosity solution of (11) (see [8]) and we claim that $u$ is in fact the maximum viscosity subsolution of $(11)$ in $C(\bar{\Omega})$. Indeed, $u$ is clearly a maximum of (73) and, for any subsolution $w \in C(\bar{\Omega})$ of $(11), z(x)=$ $\max (u(x), w(x))$ is again a subsolution of (11). But then $\int z d x \leq \int u d x$ and therefore $z \equiv u$ on $\bar{\Omega}$ and this yields $u \geq w$ on $\bar{\Omega}$.

Hence, the only thing to prove is that $\left(v_{n}\right)$ is compact in $C(\bar{\Omega})$. If (34) holds, then $\nabla v_{n}$ is bounded in $\Omega$. From the equation satisfied by $v_{n}$ it follows that $v_{n}$ is bounded in $W^{1, \infty}(\Omega)$, hence compact in $C(\bar{\Omega})$. If $(35)$ and $(\mathrm{H} 2)$ hold, then the Lipschitz norm of $v_{n}$ is bounded only in a neighborhood of $\partial \Omega$ but the same conclusion can be drawn since an a priori estimate on the modulus of continuity of $v_{n}$ near $\partial \Omega$ propagates in $\Omega$ by the methods in [10], using (H2). 
We conclude this section with another method of approximation. To simplify the presentation we will consider only the case of

$$
u+H(x, \nabla u)=0 \text { in } \Omega \text {. }
$$

The idea is to approximate the problem of finding a solution $u$ of (74) which is a viscosity supersolution on $\bar{\Omega}$ by a family of problems of the form: $u_{\varepsilon}$ is a viscosity solution of

$$
u_{\varepsilon}+H\left(x, \nabla u_{\varepsilon}\right)=f_{\varepsilon} \quad \text { in } \Omega, \quad u_{\varepsilon} \in C(\Omega),
$$

such that

$$
u_{\varepsilon}(x) \rightarrow+\infty \text { as } \operatorname{dist}(x, \partial \Omega) \rightarrow 0_{+},
$$

where $f_{\varepsilon} \in C(\Omega)$ converges to 0 uniformly on compact subsets of $\Omega$.

Theorem VII.3. Let us assume either (34) or (35) and (H2). Then there exists $f_{\varepsilon} \in C(\Omega), f_{\varepsilon} \geq 0, f_{\varepsilon} \rightarrow 0$ as $\varepsilon \rightarrow 0$, uniformly on compact subsets of $\Omega$, such that (75) has a viscosity solution $u_{\varepsilon}$ satisfying (76). Moreover, $u_{\varepsilon}$ converges uniformly on compact subsets of $\Omega$ as $\varepsilon \rightarrow 0$ to the unique viscosity solution $u \in C(\bar{\Omega})$ of (74) which is a viscosity supersolution of (74) on $\bar{\Omega}$.

Proof. Take any $\underline{w} \in C^{1}(\Omega), \underline{w} \geq 0, \underline{w}(x) \rightarrow \infty$ as $\operatorname{dist}(x, \partial \Omega) \rightarrow 0_{+}$. Let $C_{0}=\|H(x, 0)\|_{\infty}$ and set $\underline{w}_{\varepsilon}=-C_{0}+\varepsilon \underline{w}, f_{\varepsilon}=\left(H\left(x, \nabla \underline{w}_{\varepsilon}\right)+\underline{w}_{\varepsilon}\right)^{+}$. Obviously, $f_{\varepsilon} \geq 0, f_{\varepsilon} \in C(\Omega), f_{\varepsilon} \rightarrow 0$ as $\varepsilon \rightarrow 0$ uniformly on compact subsets of $\Omega$ and $\underline{w}_{\varepsilon} \in C^{1}(\Omega)$ is a subsolution of (75). We then claim there exists a viscosity solution $u_{\varepsilon} \in C(\Omega)$ of (75) satisfying $\underline{w}_{\varepsilon} \leq u_{\varepsilon}$. This claim of course implies the first part of the statement. To prove this claim, we consider the problem

$$
u_{\varepsilon}^{\delta}+H\left(x, \nabla u_{\varepsilon}^{\delta}\right)=f_{\varepsilon} \quad \text { in } \Omega_{s}, \quad u_{\varepsilon}^{\delta}=\underline{w}_{\varepsilon} \text { on } \partial \Omega_{\delta}
$$

where $\Omega_{\delta}=\{x \in \Omega, \operatorname{dist}(x, \partial \Omega)>\delta\}$. Using (34) (or (35) and (H2)) one deduces, for $\delta$ small enough, the existence of a viscosity solution $u_{\varepsilon}^{\delta} \in C(\Omega)$ of (77) from the results of P.-L. Lions [23], G. Barles [1, 2] and $u_{\varepsilon}^{\delta} \geq w_{\underline{\varepsilon}}$ on $\bar{\Omega}_{\delta}$ by standard comparison results. In fact, we even have for $\delta_{1}<\delta_{2}$

$$
u_{\varepsilon}^{\delta_{1}} \geq u_{\varepsilon}^{\delta_{2}} \geq \underline{w}_{\varepsilon} \quad \text { on } \bar{\Omega}_{\delta_{2}} .
$$

If (34) holds, we deduce from equation (77) that $\nabla u_{\varepsilon}^{\delta}$ and $u_{\varepsilon}^{\delta}$ are bounded in $L^{\infty}\left(\Omega_{\delta_{0}}\right)$ for $\delta<\delta_{0}$, for any $\delta_{0}>0$ fixed. If (35) holds, we obtain similar estimates only in $\Omega_{\delta_{0}}-\Omega_{\delta_{1}}$ for $0<\delta_{1}<\delta_{0}$ where $\delta_{0}$ is a fixed positive constant. And then using $(\mathrm{H} 2)$ as in M. G. Crandall and P.-L. Lions $[9,10]$ we obtain estimates on $u_{\varepsilon}^{\delta}$ and its modulus of continuity on each $\Omega_{\delta_{1}}$.

Therefore in both cases $u_{\varepsilon}^{\delta}$ converges uniformly on compact subsets of $\Omega$ as $\delta$ goes to 0 to some $u_{\varepsilon} \in C(\Omega)$ which is a viscosity solution of (75) and such that $u_{\varepsilon} \geq \underline{w}_{\varepsilon}$ on $\Omega$. Let us discuss now the convergence of $u_{\varepsilon}$. 
Since $f_{\varepsilon} \geq 0$ for all $\varepsilon$, we deduce easily by standard comparison results that $u_{\varepsilon} \geq-\|H(x, 0)\|_{\infty}=-C_{0}$. This combined with equation (75) easily yields as above that $u_{\varepsilon}$ is relatively compact in $C(\Omega)$ or, in other words, we may assume (extracting a sequence $\varepsilon_{n} \rightarrow 0$ if necessary) that $u_{\varepsilon}$ converges uniformly on compact subsets of $\Omega$ to some $u \in C(\Omega)$ which is a viscosity solution of (74) and $u \geq-C_{0}$. Then, still because of equation (74), $u$ in fact belongs to $C(\bar{\Omega})$ (or may be extended in $C(\bar{\Omega})$ ). To conclude we just have to prove that $u$ is a viscosity supersolution of (74) on $\bar{\Omega}$.

We first observe that $u_{\varepsilon} \geq u$ on $\Omega$. Indeed, for $\delta$ small enough (75) implies that $u_{\varepsilon} \geq u$ on $\partial \Omega_{\delta}$. Since $u_{\varepsilon}$ is a viscosity supersolution of (74) on $\Omega_{\delta}$, then, by standard comparison results, $u_{\varepsilon} \geq u$ on $\Omega_{\delta}$ and we conclude letting $\delta$ go to 0 .

Next, let $\phi \in C^{1}(\bar{\Omega})$ and let $x_{0} \in \bar{\Omega}$ be a strict global minimum over $\bar{\Omega}$ of $u-\phi$. Obviously, $u_{\varepsilon}-\phi$ has a global minimum over $\Omega$ at some point $x_{\varepsilon} \in \Omega$ and $x_{\varepsilon} \rightarrow x_{0}$ as $\varepsilon \rightarrow 0_{+}$. By construction we have

$$
u_{\varepsilon}\left(x_{\varepsilon}\right)+H\left(x_{\varepsilon}, \nabla \phi\left(x_{\varepsilon}\right)\right) \geq 0 .
$$

To conclude we need to prove that $u_{\varepsilon}\left(x_{\varepsilon}\right) \rightarrow u\left(x_{0}\right)$ as $\varepsilon \rightarrow 0$. But on one hand

$$
u_{\varepsilon}\left(x_{\varepsilon}\right) \geq u\left(x_{\varepsilon}\right) \rightarrow u\left(x_{0}\right) \quad \text { as } \varepsilon \rightarrow 0
$$

while for any $x \in \Omega$,

$$
u_{\varepsilon}\left(x_{\varepsilon}\right)-\phi\left(x_{\varepsilon}\right) \leq u_{\varepsilon}(x)-\phi(x) \rightarrow u(x)-\phi(x) \text { as } \varepsilon \rightarrow 0,
$$

hence, letting $x$ go to $x_{0}$, we find $\varlimsup_{\varepsilon} u_{\varepsilon}\left(x_{\varepsilon}\right) \leq u\left(x_{0}\right)$ and we conclude.

\section{ERGODIC PROBLEMS}

As in the preceding section we consider here a Hamiltonian $H(x, p) \in$ $C\left(\bar{\Omega} \times R^{N}\right)$ where $\Omega$ is (to simplify) a bounded smooth open domain in $R^{N}$ and we are interested in the so-called ergodic problems associated with $H$. More precisely, by the results of $\S \S I I I$ and $\mathrm{V}$ we know that if (37) holds, then for any $u_{0} \in C(\bar{\Omega})$ there exist viscosity solutions $u_{\varepsilon} \in C(\bar{\Omega})(u \in C(\bar{\Omega}) \times[0, T])$ $(\forall T<\infty)$ of

$$
\begin{gathered}
H\left(x, \nabla u_{\varepsilon}\right)+\varepsilon u_{\varepsilon}=0 \quad \text { in } \Omega, \\
\frac{\partial u}{\partial t}+H(x, \nabla u)=0 \quad \text { in } \Omega \times(0, \infty),\left.\quad u\right|_{t=0}=u_{0} \quad \text { in } \bar{\Omega},
\end{gathered}
$$

which are in addition viscosity supersolution on $\bar{\Omega},(\bar{\Omega} \times(0, \infty)$, respectively). In this section we consider the problem of the asymptotic behavior of $u_{\varepsilon}$, $u(\cdot, t)$ as $\varepsilon \rightarrow 0_{+}, t \rightarrow+\infty$, respectively. Related ergodic problems have been investigated by M. Robin [30, 31], A. Bensoussan [4], F. Gimbert [14], I. Capuzzo-Dolcetta and M. G. Garroni [5], I. Capuzzo-Dolcetta and J. L. Menaldi [34], J. M. Lasry and P.-L. Lions [22], P.-L. Lions and B. Perthame [28], P.-L. Lions [25, 26], P.-L. Lions, G. Papanicolau and S. R. S. Varadhan [27]. Our main result is 
Theorem VIII.1. We assume (34). Let $u_{0} \in C(\bar{\Omega})$.

(i) There exists a unique $\lambda \in R$, such that there exists $v \in C(\bar{\Omega})$ viscosity solution of

$$
H(x, \nabla v)+\lambda=0 \quad \text { in } \Omega
$$

which is a viscosity supersolution of $(70)$ on $\bar{\Omega}$.

(ii) For any $x_{0} \in \bar{\Omega}, u_{\varepsilon}(\cdot)-u_{\varepsilon}\left(x_{0}\right)$ is bounded in $W^{1, \infty}(\Omega)$ for $\varepsilon \geq 0$. Moreover, $\varepsilon u_{\varepsilon}$ converges uniformly on $\bar{\Omega}$ to $\lambda$. Finally, if $u_{\varepsilon_{n}}-u_{\varepsilon_{n}}\left(x_{0}\right)$ converges in $C(\bar{\Omega})$ to some $v$, for some sequence $\varepsilon_{n} \rightarrow 0$, then $v$ is a viscosity solution of (80) and a viscosity supersolution on $\bar{\Omega}$.

(iii) For any $x_{0} \in \bar{\Omega}, u(\cdot, t)-u\left(x_{0}, t\right)$ is relatively compact in $C(\bar{\Omega})$ for $t \geq 0$ and $\frac{1}{t}(u(\cdot, t))$ converges uniformly on $\bar{\Omega}$ to $\lambda$.

Proof. The proof is almost identical to the corresponding one in P.-L. Lions [25]. Indeed, by comparison results $\left|\varepsilon u_{\varepsilon}\right|<\|H(x, 0)\|_{\infty}$ and thus by (78) and (34) $\nabla u_{\varepsilon}$ is bounded in $L^{\infty}(\Omega)$. Therefore, up to subsequences we may assume that $u_{\varepsilon}-u_{\varepsilon}\left(x_{0}\right)$ converges uniformly on $\bar{\Omega}$ to some $v \in C(\bar{\Omega}), \varepsilon u_{\varepsilon}$ converges uniformly on $\bar{\Omega}$ to $\lambda \in R$ and obviously $v$ is a viscosity solution of (70) and a viscosity supersolution on $\bar{\Omega}$. We claim that $\lambda$ is unique. Indeed, if $\mu_{1} \leq \mu_{2}$ are two constants such that there exists $v_{1}, v_{2}$ associated with $\mu_{1}$, $\mu_{2}$ as above, then let $C_{0}>\left\|v_{1}-v_{2}\right\|_{\infty}$; we obviously have for $\delta$ small enough

$$
-\mu_{1}+\delta v_{1} \geq-\mu_{2}+\delta\left(v_{2}+C_{0}\right) \text { on } \bar{\Omega},
$$

and since $v_{1}, v_{2}+C_{0}$ are respectively viscosity solutions in $\Omega$ and supersolutions in $\bar{\Omega}$ of

$$
\begin{aligned}
& H\left(x, \nabla v_{1}\right)+\delta v_{1}=-\mu_{1}+\delta v_{1}, \\
& H\left(x, \nabla\left(v_{2}+C_{0}\right)\right)+\delta\left(v_{2}+C_{0}\right)=-\mu_{2}+\delta\left(v_{2}+C_{0}\right),
\end{aligned}
$$

therefore by comparison results, $v_{2}+C_{0} \leq v_{1}$. The contradiction proves the uniqueness of $\lambda$. Hence, (i) and (ii) are proved.

To prove (iii), recalling that the map $u_{0} \rightarrow u(\cdot, t)$ is a contraction in $C(\bar{\Omega})$, we see that we may assume that $u_{0} \in W^{1, \infty}(\Omega)$. In which case one proves by standard methods, using the equation and (34), that $\nabla u_{\varepsilon}, \partial u_{\varepsilon} / \partial t$ are bounded in $L^{\infty}(\Omega \times(0, \infty))$. To conclude one considers a constant $C_{0}$ such that

$$
v(x)+C_{0} \geq u_{0}(x) \geq v(x)-C_{0} \text { on } \bar{\Omega}
$$

and one deduces easily in view of the equation satisfied by $v$

$$
v(x)+C_{0}+\lambda t \geq u(x, t) \geq v(x)-C_{0}+\lambda t .
$$

The proof is complete dividing by $t$ and letting $t$ go to $\infty$.

Remark. Of course, if $v$ solves (80), $v+c$ also solves (80) for all $c \in R$. But in fact, even up to the addition of a constant $c \in R, u$ is not the unique solution of (80) and a supersolution on $\bar{\Omega}$. Indeed, consider the example

$$
H(x, p)=(|p|-1)^{+} \text {. }
$$


Then, one checks easily that, taking $\lambda=0$ and $v$ to be any Lipschitz function on $\Omega$ with $|\nabla v| \leq 1$ a.e. on $\Omega, v$ is indeed a viscosity solution of (80) in $\Omega$ and a viscosity supersolution on $\bar{\Omega}$.

\section{MiXed BOUNDARY CONDITIONS}

In this section, we want to consider more general boundary conditions than the ones studied in the preceding sections. For example, we wish to consider viscosity solutions $u \in C(\bar{\Omega})$ of (1) which are viscosity supersolutions of (1) on $\Omega \cup \Gamma_{1}$ where $\Gamma_{1}$ is a relatively open subset of $\partial \Omega$, while on the complement $\Gamma_{0}=\partial \Omega-\Gamma_{1} u$ takes (for example) Dirichlet boundary conditions, i.e. $u$ is given on $\Gamma_{0}$. As before we will consider the two model cases (11) and (12). Observe by the way, that in the case of (12), since we prescribe initial conditions in all existence and uniqueness results, we already considered a particular case of this problem where $\Gamma_{0}=\partial \Omega \times\{0\}$. Therefore, in the case of (11) we will prescribe $u$ on a closed set $\Gamma_{0}$ of $\partial \Omega$, while in (12) we will prescribe $u$ on a closed subset $\Gamma_{0}$ of $\partial \Omega \times[0, T]$ (in such a way that this boundary condition matches with the initial condition on $\bar{\Omega} \times\{0\}$ ). Of course, all the elementary results described in $\S I I$ still hold in this context.

We will be mainly interested in existence and uniqueness results which basically are very much of the same kind of those obtained above. This is way we will only present the analogues of Theorems III.1, III.2 and V.1, V.2, explaining the main changes in the proof given before. We begin with uniqueness results for (11) analogous to Theorems III.1, III.2. To simplify, we take $\Omega$ bounded.

Theorem IX.1. Let $\Gamma_{0}$ be a nonempty closed subset of $\partial \Omega$. Assume that $(\mathrm{H} 1)$ holds. Let $u, v \in C(\bar{\Omega})$ be respectively a viscosity subsolution of (11) in $\Omega$ and a viscosity supersolution of

$$
v+H(x, v, \nabla v)+f(x)=0 \quad \text { in } \Omega \cup \Gamma_{1}
$$

where $f \in C(\bar{\Omega})$. Then, if either $(\mathrm{H} 2)$ or $(\mathrm{H} 3)$ hold or $u$ is Lipschitz, we have

$$
\max _{\bar{\Omega}}(u-v)^{+} \leq \max \left\{\max _{\bar{\Omega}} f^{+}, \max _{\Gamma_{0}}(u-v)^{+}\right\} .
$$

Theorem IX.2. Let $\Gamma_{0}$ be a nonempty closed subset of $\partial \Omega \times[0, T]$. Assume that $(\mathrm{H} 1)$ holds. Let $u, v \in C(\bar{\Omega} \times(0, T))$ be respectively a viscosity solution of (12) and a viscosity supersolution of

$$
\frac{\partial v}{\partial t}+H(x, t, v, \nabla v)+f(x, t)=0 \quad \text { in } \Omega \times(0, T) \cup \Gamma_{1}
$$

where $f \in C(\bar{\Omega} \times[0, T))$. Then, if either $(\mathrm{H} 2)$ or $(\mathrm{H} 3)$ hold or $u$ is Lipschitz in $x$, uniformly for $t \in(0, T]$, we have for all $t \in(0, T]$

$$
\underset{\bar{\Omega} \times[0, T]}{\operatorname{Max}}(u-v)^{+} \leq \operatorname{Max}\left\{\operatorname{Max}_{\bar{\Omega}}(u-v)^{+}(\cdot, 0)+\int_{0}^{t} \max _{\bar{\Omega}} f^{+}(\cdot, s) d s ; \max _{\Gamma_{0}}(u, v)^{+}\right\} .
$$


Remarks. (i) Assumption (H3) is actually needed to hold only in a neighborhood of $\Gamma_{1}$.

(ii) The comparison inequality (82) holds also under the assumptions $(\mathrm{H} 1)$, (H2), (H4) (respectively, (H1), (28), (29) or (H1), (H2), (28), (33)). These claims can be verified following the line of the proof of Theorem III.5 (Theorem III.6 or III.7, respectively). The proofs of Theorems IX.1, IX.2 are analogous (with the usual adaptations) and therefore we prove Theorem IX.1 only.

Proof of Theorem IX.1. Let $\alpha>0$ and consider $\chi_{\alpha} \in C^{1}(\bar{\Omega})$ such that $\chi_{\alpha}(x)=$ 0 if $\operatorname{dist}\left(x, \Gamma_{0}\right)<\alpha, \chi_{\alpha}(x)=1$ if $\operatorname{dist}\left(x, \Gamma_{0}\right)>2 \alpha$ and $0 \leq \chi_{\alpha} \leq 1$. At this point we follow the proof of Theorem III.1 introducing, the same notations therein,

$$
w_{\varepsilon}^{\alpha}(x, y)=u(x)-v(y)-\frac{1}{\varepsilon^{2}}\left|x+\varepsilon \chi_{\alpha}(x) T(x)-y\right|^{2} .
$$

We prove (82) by contradiction. Therefore, fixing $\alpha>0$ small enough we may assume that there exists $\gamma>0$ such that

$$
\max _{\bar{\Omega}}(u-v) \geq \gamma+\max \mid \max _{\bar{\Omega}} f^{+}, \quad \max \left\{(u-v)^{+}(y), \operatorname{dist}\left(y, \Gamma_{0}\right)\right\} \leq 2 \alpha .
$$

As in Theorem III.1, one easily checks that

$$
\max _{\Omega \times \Omega} w_{\varepsilon}^{\alpha}(x, y) \geq \max _{\bar{\Omega}}(u-v)-\omega(\varepsilon)
$$

for some modulus $\omega$. In addition, if $(x, y) \in \bar{\Omega} \times \bar{\Omega}$ is a maximum point of $w_{\varepsilon}^{\alpha}$ on $\bar{\Omega} \times \bar{\Omega}$, we have

$$
w_{\varepsilon}^{\alpha}(\bar{x}, \bar{y}) \leq \max _{\bar{\Omega}}(u-v)+\omega(|\bar{x}-\bar{y}|)-\frac{1}{\varepsilon^{2}}\left|\bar{x}+\varepsilon \chi_{\alpha} T(\bar{x})-\bar{y}\right|^{2}
$$

and in particular we deduce for some $C \geq 0|\bar{x}-\bar{y}| \leq C \varepsilon$. Therefore, for $\varepsilon$ small enough, this yields

$$
\operatorname{dist}\left(\bar{x}, \Gamma_{0}\right)>2 \alpha, \quad \chi_{\alpha}(\bar{x})=1,
$$

and we may now argue exactly as in Theorem III.1.

Let us also mention the following uniqueness result for the problem

$$
H(x, u, \nabla u)=0 \text { in } \Omega .
$$

This case is treated by the same methods as in M. G. Crandall and P.-L. Lions [8] and $\mathrm{H}$. Ishii [20] and we skip its proof.

Proposition IX.3. We assume that $H(x, t, p)$ is convex in $p$ for all $x \in \bar{\Omega}$, $t \in R$ and that there exists $\underline{u} \in C^{1}(\bar{\Omega})$ such that

$$
H(x, \underline{u}(x), \nabla \underline{u}(x))<0 \text { in } \bar{\Omega} .
$$

Let $u, v \in C(\bar{\Omega})$ be, respectively, a viscosity subsolution of (85) and a viscosity supersolution of (85) on $\Omega \cup \Gamma_{1}$. Assume furthermore that either (H1) holds and either (H2), (H3) holds or that $u$ is Lipschitz. Then we have

$$
\max _{\bar{\Omega}}(u-v)^{+} \leq \max _{\Gamma_{0}}(u-v)^{+} .
$$


We now turn to existence results which are analogous to Theorems IV.1 and IV.2. In the case of the stationary problem we will consider the following problem:

$$
\lambda u+H(x, u, \nabla u)=0 \text { in } \Omega
$$

with $\lambda \geq 0$. We will assume as in P.-L. Lions [23] and G. Barles [1, 2] that there exists

$$
\underline{u} \in C(\bar{\Omega}), \underline{u} \text { is a viscosity subsolution of }(78), \underline{u}=\phi \text { on } \Gamma_{0}
$$
where $\phi$ is given.

Theorem IX.4. We assume (H1), (89) and either (34), or (35), (H2) and $\lambda>0$. Then there exists $u \in C(\bar{\Omega})$ viscosity solution of (88) such that $u=\phi$ on $\Gamma_{0}$ and $u$ is a viscosity supersolution of (89) on $\Omega \cup \Gamma_{1}$.

Remark. If $\lambda=0$ (when (34) holds) and if $H(x, p)$ is not convex in $p$, we do not have uniqueness, in general, of such solutions (see [23] for related examples). But in fact the proof below shows that if $\lambda=0$ and (34) holds there exists a maximum viscosity subsolution with the above properties.

In the case of the Cauchy problem (12), we will need to assume that there exists $\underline{u} \in C(\bar{\Omega} \times[0, T])$ such that

$$
\underline{u} \text { is a viscosity subsolution of }(12),\left.\underline{u}\right|_{t=0}=u_{0} \text { in } \bar{\Omega},\left.\underline{u}\right|_{\Gamma}=\phi
$$
where $u_{0}, \phi$ are given initial and boundary conditions respectively.

Theorem IX.5. We assume (H1), (90), (36) and either (34), or (35) and (H3). Then, there exists a unique viscosity solution $u \in C(\bar{\Omega} \times[0, T])$ of (12) such that $\left.u\right|_{t=0}=u_{0}$ on $\bar{\Omega},\left.u\right|_{\Gamma}=\phi$ and $u$ is a viscosity supersolution of (12) on $(\bar{\Omega} \times[0, T]) \cup \Gamma_{1}$.

Let us just explain the proof of Theorem IX.4 in the case when (34) holds.

To simplify the presentation even more we assume that $H=H(x, p)$. We then argue as in $\S$ VII: we build a nondecreasing sequence of viscosity solutions $\left(u_{n}\right)_{n \geq 1}$ of (88) maximizing asymptotically $\int_{\Omega} v d x$ over all viscosity subsolutions of (88) which are less than $\phi$ on $\Gamma_{0}$, and such that $u_{n}=\phi$ on $\Gamma_{0}$. Indeed, using the results of P.-L. Lions [23] and G. Barles [1,2] we see that we can adapt the arguments of $\S \mathrm{VII}$ to our case here. And one proves as in $\S \mathrm{VII}$ that $u_{n}$ converges on $C(\bar{\Omega})$ to the maximum viscosity solution $u$ (or subsolution) of (88) such that $u=\phi$ on $\Gamma_{0}$ (or $u \leq \phi$ on $\Gamma_{0}$ ). Finally, the fact that $u$ is a viscosity supersolution of (88) on $\Omega \cup \Gamma_{1}$ follows from the results of $\S I I$.

\section{SOME APPLICATIONS}

We present in this section some applications of the results proved in the preceding sections to calculus of variations and optimal control theory. We begin with the study of some distance-like functions and related minimization problems. Roughly speaking, we will consider minimization problems over curves which are restricted to stay in a given domain. The restriction (or constraint) 
will in fact lead to a completely similar treatment of such problems as in P.-L. Lions [23], provided one now uses viscosity solutions which are supersolutions on the closure of the domain.

We consider some Hamiltonian $H(x, p) \in C\left(\bar{\Omega} \times R^{N}\right)$ convex in $p$ for all $x \in \bar{\Omega}$, where $\Omega$ is a given bounded (for instance) smooth open domain in $R^{N}$. We denote by $L(x, q)$ the associated Lagrangian

$$
L(x, q)=\sup _{p \in R^{N}}\{(p, q)-H(x, p)\} \leq+\infty
$$

and we will always assume

$$
H(x, p) \rightarrow+\infty \text { as }|p| \text { is } \infty, \quad \text { uniformly for } x \in \bar{\Omega} .
$$

We now introduce various variational problems. We begin with problems involving terminal constraints: Let $x, y \in \bar{\Omega}, \lambda>0, T>0$,

$$
\begin{array}{r}
d(x, y)=\inf \left\{\int_{0}^{t} L(\xi(s), \dot{\xi}(s)) d s \mid \xi \in C^{1}(0, t ; \bar{\Omega}), \xi(0)=y,\right. \\
\xi(t)=x \text { for some } t \geq 0\},
\end{array}
$$

$$
d_{\lambda}(x)=\inf \left\{\int_{0}^{\infty} L(\xi(s),-\dot{\xi}(s)) \bar{e}^{\lambda s} d s \mid \xi \in C^{1}(0, \infty ; \bar{\Omega}), \xi(0)=x\right\}
$$

$$
d_{0}(x)=\inf \left\{\frac{\lim }{T \rightarrow \infty} \frac{1}{T} \int_{0}^{T} L(\xi(s), \dot{\xi}(s)) d s \mid \xi \in C^{1}(0, \infty ; \bar{\Omega}), \xi(0)=x\right\}
$$

$$
\begin{gathered}
u(x)=\inf _{y \in \partial \Omega}(d(x, y)+\phi(y)), \\
u_{\lambda}(x)=\inf \left\{\int_{0}^{T} L(\xi(s),-\dot{\xi}(s)) e^{-\lambda s} d s+\phi(\xi(T)) e^{-\lambda T} \mid\right. \\
\left.\xi \in C^{1}(0, T ; \Omega), \xi(0)=x, \xi(T) \in \partial \Omega \text { for some } T \geq 0\right\},
\end{gathered}
$$

$$
\begin{array}{r}
d(x, y, T)=\inf \left\{\int_{0}^{T} L(\xi(s), \dot{\xi}(s)) d s / \xi \in C^{1}(0, T ; \bar{\Omega}), \xi(0)=y,\right. \\
\xi(T)=x\},
\end{array}
$$

$$
\begin{array}{r}
u(x, T)=\inf \left\{\int_{0}^{T} L(\xi(s),-\dot{\xi}(s)) d s+h(\xi(T)) / \xi \in C^{1}(0, T ; \bar{\Omega})\right. \\
\xi(0)=x, \xi(T)=y\},
\end{array}
$$


where $\phi$ is any l.s.c. function on $\partial \Omega$ taking values in $R \cup\{+\infty\}$ bounded from below with $\phi \not \equiv+\infty$ and $h$ is any l.s.c. function on $\bar{\Omega}$ taking values in $R \cup\{+\infty\}$ bounded from below. We will also use the following assumption:

$$
H(x, p)|p|^{-1} \rightarrow+\infty \text { as }|p| \rightarrow \infty, \quad \text { uniformly for } x \in \bar{\Omega} .
$$

Our main result is

Theorem X.1. Let $H(x, p) \in C\left(\bar{\Omega} \times R^{N}\right)$ be convex in $p$ for all $x \in \bar{\Omega}$ and satisfy (92).

(1) For all $y \in \bar{\Omega}$, the function $d(\cdot, y)$ given by (93) is Lipschitz on $\bar{\Omega}, a$ viscosity solution of

$$
H(x, \nabla u)=0 \quad \text { in } \Omega-\{y\}, \quad u(y)=0,
$$

and a viscosity supersolution of (101) on $\bar{\Omega}-\{y\}$. Furthermore, $d(0, y)$ is the maximum viscosity subsolution in $C(\bar{\Omega})$ of

$$
H(x, \nabla v)=0 \quad \text { in } \Omega
$$

such that $v(y) \leq 0$. Finally, if $(86)$ holds, $d(\cdot, y)$ is the unique viscosity solution of (100) which is a viscosity supersolution on $\bar{\Omega}-\{y\}$.

(2) For $\lambda>0$, the function $d_{\lambda}(\cdot)$ given by (94) is Lipschitz on $\bar{\Omega}$ and is the unique viscosity solution in $\Omega$ and supersolution on $\bar{\Omega}$ of

$$
\lambda u+H(x, \nabla u)=0 \quad \text { in } \Omega, \quad u \in C(\bar{\Omega}) .
$$

(3) The function $d_{0}(\cdot)$ given by (95) is the unique constant $\bar{\lambda}$ such that there exists a viscosity solution in $\Omega$ and supersolution on $\bar{\Omega}$ of

$$
\bar{\lambda}+H(x, \nabla v)=0 \quad \text { in } \Omega, \quad v \in C(\bar{\Omega}) .
$$

Furthermore, $\lambda d_{\lambda}(x)$ converges uniformly on $\bar{\Omega}$ to $d_{0}$ as $\lambda$ goes to $0_{+}$and $\frac{1}{T} d(x, y, T)$ converges uniformly for $x, y \in \bar{\Omega}$ to $d_{0}$, as $T$ goes to $+\infty$.

(4) The function $u$ (resp. $u_{j}$ ) given by (96) (resp. (97)) is Lipschitz on $\bar{\Omega}$ and is the maximum viscosity subsolution in $C(\bar{\Omega})$ of (102) (resp. (103)) such that $v \leq \phi$ on $\partial \Omega$. The set $\{x \in \partial \Omega / u(x)=\phi(x)\} \quad\left(\right.$ resp. $u_{\lambda}(x)=\phi(x)$ ) is closed and we denote it by $\Gamma_{0}$ while we denote by $\Gamma_{1}$ its complement in $\partial \Omega$. In addition, $u$ is the maximum (resp. unique) viscosity solution in $C(\bar{\Omega})$ of (102) (resp. (103)) such that $u=\phi$ on $\Gamma_{0}$ (resp. $u_{\lambda}=\phi$ on $\left.\Gamma_{0}\right)$ and $u$ (resp. $u_{\lambda}$ ) is a viscosity supersolution of (102) (resp. (103)) on $\Omega \cup \Gamma_{1}$. Furthermore, if there exists a viscosity subsolution $v$ of (102) (resp. (103)) such that $v \in C(\bar{\Omega})$, $v=\phi$ on a closed nonempty subset $\tilde{\Gamma}_{0}$ of $\partial \Omega$ while $\phi \equiv+\infty$ on $\partial \Omega-\tilde{\Gamma}_{0}$ then $\Gamma_{0}=\tilde{\Gamma}_{0}$. Finally, if (84) holds, $u$ is the unique viscosity solution in $C(\bar{\Omega})$ of (102) such that $u=\phi$ on $\Gamma_{0}$ and $u$ is a viscosity supersolution of (102) on $\Omega \cup \Gamma_{1}$.

(5) If we assume (100), the function $u(x, t)$ given by (99) is Lipschitz on $\bar{\Omega} \times(0, \infty)($ for all $\varepsilon>0)$, l.s.c. on $\bar{\Omega} \times[0, \infty[$ and is the unique viscosity solution in $C(\bar{\Omega} \times(\varepsilon, \infty))(\forall \varepsilon>0)$ of

$$
\partial u / \partial t+H(x, \nabla u)=0 \quad \text { in } \Omega \times(0, \infty)
$$


which is a viscosity supersolution of $(95)$ on $\bar{\Omega} \times(0, \infty)$ and such that

$$
u(x, t) \rightarrow h(x) \text { as } t \rightarrow 0_{+}, \quad \text { for all } x \in \bar{\Omega} .
$$

(ii) It is quite obvious that (98) is a special case of (99) choosing $h(x)=$ $1_{\{y\}}=0$ if $x=y,=+\infty$ if $x \neq y$.

Proof. (1) Most of part (1) is proved in P.-L. Lions [23] and one deduces the fact that $d(\cdot, y)$ is a viscosity supersolution of (104) on $\Omega-\{y\}$ by either a direct verification argument or by using the results of $\S I$. Then, the uniqueness follows from Proposition IX.3.

(2) is proved similarly and (3) is deduced from (2) by the results of $\S$ VIII. To prove part (4) we just have to prove that $u$ is a viscosity supersolution of (102) on $\Omega \cup \Gamma_{1}$. Indeed, the remainder follows upon combining the results of P.-L. Lions [23], this fact and the results of the preceding sections (one argues similarly for $u_{\lambda}$ ). Observe also that $\Gamma_{0}$ is closed since $u \leq \phi$ on $\partial \Omega$ while $u-\phi$ is u.s.c. To show that it is viscosity supersolution of (102) on $\Omega \cup \Gamma_{1}$, we observe that we know from [23] that it is the maximum viscosity subsolution of (102) with $u \leq \phi$ on $\partial \Omega$. Hence, near a point $x_{0}$ of $\partial \Omega$ where $u\left(x_{0}\right)<\phi\left(x_{0}\right)$ we may argue as in $\S I I$ to deduce from this fact the property we claimed.

Finally, (5) is deduced from the results of P.-L. Lions [23] and, for example, the observations made in $\S I I$, while the uniqueness is obtained by convenient combinations of the arguments made above and of those developed in M. G. Crandall, P.-L. Lions and P. E. Souganidis [12].

We now turn to optimal control problems. We begin with infinite horizon problems. As before, everything we say will concern (to simplify) the case of a bounded, smooth open region $\Omega$ of $R^{N}$. We first define the state equations

$$
\dot{X}_{t}=b\left(X_{t}, \alpha_{t}\right) \quad \text { for } t \geq 0, \quad X_{0}=x \in \bar{\Omega}
$$

where $\alpha_{t}$ (the control process) is a measurable function from $(0, \infty)$ into a given metric space $A$. We assume $b$ satisfies

$$
|b(x, \alpha)| \leq C, \quad|b(x, \alpha)-b(y, \alpha)| \leq C|x-y|, \quad \forall x, y \in R^{N}, \forall \alpha \in A
$$

for some $C \geq 0$ and $b$ is continuous on $R^{N} \times A$. We will impose stateconstraints on our control problem, i.e. we will restrict our attention to control $\alpha_{t}$ in the following class of admissible controls $\mathscr{A}_{x}$ (depending on the initial position $x$ ):

$$
\mathscr{A}_{x}=\left\{\alpha_{t} / X_{t} \in \bar{\Omega} \text { for all } t \geq 0\right\} .
$$

We now consider a cost function for $x \in \bar{\Omega}, \alpha_{t} \in \mathscr{A}_{x}$ :

$$
J\left(x, \alpha_{t}\right)=\int_{0}^{\infty} f\left(X_{t}, \alpha_{t}\right) e^{-\lambda t} d t
$$

where $\lambda>0$ (discount factor), $f$ (the running cost) will be always assumed to satisfy

$$
|f(x, \alpha)| \leq C, \quad|f(x, \alpha)-f(y, \alpha)| \leq \omega(|x-y|), \quad \forall x, y \in R^{N}, \forall \alpha \in A
$$


for some modulus $\omega$ and for some constant $C \geq 0$. We define the value function of this infinite horizon control problem with state constraints by

$$
u(x)=\inf _{x, \in \mathscr{X}_{*}} J\left(x, \alpha_{t}\right), \quad \forall x \in \bar{\Omega} .
$$

We will use the following assumptions:

$$
\begin{gathered}
\exists \nu>0, \forall x \in \partial \Omega, \alpha \in A, \quad b(x, \alpha) \cdot n(x) \leq-\nu<0, \\
\exists C \geq 0, \forall x, y \in R^{N}, \forall \alpha \in A, \quad|f(x, \alpha)-f(y, \alpha)| \leq C|x-y| .
\end{gathered}
$$

Our main result is

Theorem X.2. We assume (112). Then $u \in C(\bar{\Omega})$ is the unique viscosity solution in $\Omega$ and supersolution on $\bar{\Omega}$ of

$$
\lambda u+\sup _{\alpha \in A}[-b(x, \alpha) \cdot \nabla u(x)-f(x, \alpha)]=0 \quad \text { in } \Omega .
$$

Furthermore, if (113) holds, $u \in C^{0, \theta}(\bar{\Omega})$ where $\theta=\lambda / \lambda_{0}$ if $\lambda<\lambda_{0}, \theta$ is arbitrary in $(0,1)$ if $\lambda=\lambda_{0}, \theta=1$ if $\lambda>\lambda_{0}$ and $\lambda_{0}$ is a fixed positive constant depending only on $b$ and $\Omega$. Finally, if the following holds:

$$
\exists \mu>0, \quad \overline{\operatorname{co}}\{b(x, \alpha) / \alpha \in A\} \supset B_{\mu} \text { for all } x \in \bar{\Omega},
$$

then $\lambda u$ converges uniformly on $\bar{\Omega}$ to the unique constant $\bar{u} \in R$ such that there exists $v \in C(\bar{\Omega})$ viscosity solution in $\Omega$ and supersolution on $\bar{\Omega}$ of

$$
\bar{u}+\sup _{\alpha \in A}[-b(x, \alpha) \cdot \nabla v-f(x, \alpha)]=0 \text { in } \Omega .
$$

Proof. In M. H. Soner [32], the first part of this result is proved. Using the examples given in $\S \mathrm{V}$ and the existence results of $\S \mathrm{VI}$ we deduce the existence of a viscosity solution in $C^{0, \theta}(\bar{\Omega})$ of (114) which is a viscosity supersolution of (114) on $\bar{\Omega}$. Next, if (115) holds we observe that

$$
H(x, p)=\sup _{\alpha \in A}[-b(x, \alpha) \cdot-f(x, \alpha)]
$$

satisfies

$$
H(x, p) \geq \mu|p|-C \quad \text { for all }(x, p) \in \bar{\Omega} \times R^{N} .
$$

Then, one completes the proof of Theorem X.2 applying Theorem VIII.1.

We continue with a result concerning finite horizon problems: we just give the setting and state the main results which can be deduced from the results of the preceding sections. Let $T<\infty$, for $x \in \bar{\Omega}, t \in[0, T]$ we consider the solutions of

$$
\dot{X}_{s}=b\left(X_{s}, s, \alpha_{s}\right) \text { for } s \geq t, X_{t}=x
$$

where $\alpha_{s}$ (measurable functions from $[t, T]$ into $A$ ) is admissible if $X_{s} \in \bar{\Omega}$ for $t \leq s \leq T$. We will denote this by $\alpha_{s} \in \mathscr{A}_{x, t}$. We assume that $b$ is continuous on $R^{N} \times[0, T] \times A$ and that

$$
\begin{array}{r}
|b(x, t, \alpha)| \leq C, \quad|b(x, t, \alpha)-b(y, t, \alpha)| \leq C|x-y|, \\
\forall x, y \in R^{N}, \forall t \in[0, T], \forall \alpha \in A .
\end{array}
$$


We then set for $x \in \bar{\Omega}, t \in[0, T], \alpha_{s} \in \mathscr{A}_{x, t}$

$$
J\left(x, t, \alpha_{s}\right)=\int_{t}^{T} f\left(X_{s}, s, \alpha_{s}\right) d s+g\left(X_{T}\right)
$$

where $g \in B U C\left(R^{N}\right), f$ is continuous on $R^{N} \times[0, T] \times A$ and

$$
\begin{aligned}
&|f(x, t, \alpha)| \leq C, \quad|f(x, t, \alpha)-f(y, t, \alpha)| \leq \omega(|x-y|), \\
& \forall x, y \in R^{N}, \forall t \in[0, T], \forall \alpha \in A .
\end{aligned}
$$

We next introduce the value function

$$
u(x, t)=\inf _{\alpha_{s} \in \phi_{x, l}} J\left(x, t, \alpha_{s}\right) \quad \text { for } x \in \bar{\Omega}, t \in[0, T] .
$$

We will use the following assumptions:

$$
\begin{aligned}
& \exists \nu>0, \forall x \in \partial \Omega, \forall t \in[0, T], \exists \alpha \in A, \quad b(x, t, \alpha) \cdot n(x) \leq-\nu, \\
& \exists C \geq 0, \forall x, y \in \bar{\Omega}, \forall \alpha \in A, \\
& \qquad|f(x, \alpha)-f(y, \alpha)|+|g(x)-g(y)| \leq C|x-y| .
\end{aligned}
$$

We then have

Theorem X.3. We assume (122). Then $u(x, t) \in C(\bar{\Omega} \times[0, T])$ is the unique viscosity solution in $\Omega \times(0, T)$ and viscosity supersolution on $\bar{\Omega} \times(0, T)$ of

$$
\begin{aligned}
& -\frac{\partial u}{\partial t}+\sup _{\alpha \in A}[-b(x, t, \alpha) \cdot \nabla u-f(x, t, \alpha)]=0 \quad \text { in } \Omega \times(0, T), \\
& \left.u\right|_{t=T}=g \text { on } \bar{\Omega} .
\end{aligned}
$$

Furthermore, if (123) holds $u$ is Lipschitz on $\Omega \times[0, T]$. Finally if $b, f$ are independent of $t$ and (115) holds, $\frac{1}{T} u(x, 0)$ converges uniformly on $\bar{\Omega}$ to $\bar{u}$ given in Theorem X.2 as $T$ goes to $+\infty$ and

$$
\bar{u}=\inf _{\alpha_{t} \in \mathscr{A}_{x}}\left\{\underline{\lim } \frac{1}{T \rightarrow \infty} \int_{0}^{T} f\left(X_{t}, \alpha_{t}\right) d t\right\} .
$$

We conclude with a result which is a direct application of the results of $\S$ VII. We consider a penalty term $p$ as in $\S$ VII and we find that if we denote by

$$
u_{\varepsilon}(x)=\inf _{\alpha_{t}} \int_{0}^{\infty}\left\{f\left(X_{t}, \alpha_{t}\right)+\frac{1}{\varepsilon} p\left(X_{t}\right)\right\} e^{-\lambda t} d t, \quad \forall x \in R^{N}
$$

or

$$
\begin{array}{r}
u_{\varepsilon}(x, t)=\inf _{\alpha_{s}}\left\{\int_{t}^{T}\left\{f\left(X_{s}, s, \alpha_{s}\right)+\frac{1}{\varepsilon} p\left(X_{s}\right)\right\} e^{-\lambda s} d s+g\left(X_{T}\right) e^{-\lambda T}\right\}, \\
\forall x \in R^{N}, \forall t \in[0, T],
\end{array}
$$

then $u_{\varepsilon}$ converges uniformly on $\bar{\Omega}$ or $\bar{\Omega} \times[0, T]$ to $u$ if (112) or (122) hold. 


\section{REFERENCES}

1. G. Barles, Existence results for first-order Hamilton-Jacobi equations, Ann. Inst. H. Poincaré Anal. Non Linéaire 1 (1984), 325-340.

2. __ Remarques sur des résultats d'existence pour les equations de Hamilton-Jacobi du premier ordre, Ann. Inst. H. Poincaré Anal. Non Linéaire 2 (1985), 21-33.

3. G. Barles and P.-L. Lions, in preparation.

4. A. Bensoussan, Méthodes de perturbation en controle optimal (to appear).

5. I. Capuzzo-Dolcetta and M. G. Garroni, Oblique derivative problems and invariant measures, Ann. Scuola Norm. Sup. Pisa Ser. (4) 13 (1986), 689-720; announced in C. R. Acad. Sci. Paris Sér. I Math. 299 (1984).

6. M. G. Crandall, L. C. Evans and P.-L. Lions, Some properties of viscosity solutions of Hamilton-Jacobi equations, Trans. Amer. Math. Soc. 262 (1984), 487-502.

7. M. G. Crandall, H. Ishii and P.-L. Lions, Uniqueness of viscosity solutions revisited.

8. M. G. Crandall and P.-L. Lions, Viscosity solutions of Hamilton-Jacobi equations, Trans. Amer. Math. Soc. 277 (1983), 1-42; announced in C. R. Acad. Sci. Paris Sér. I Math. 292 (1981), 183-186.

9. __ On existence and uniqueness of solutions of Hamilton-Jacobi equations, Nonlinear Anal. (1985).

10. __ Hamilton-Jacobi equations in infinite dimensions. Parts I, II, III, J. Funct. Anal. 62 (1985), 379-396; 65 (1986), 368-495; 68 (1986), 214-247; announced in C. R. Acad Sci. Paris Sér. I Math. 300 (1985), 67-70.

11. __ Remarks on the existence and uniqueness of unbounded viscosity solutions of HamiltonJacobi equations, Illinois J. Math. 31 (1987), 665-688; announced in C. R. Acad. Sci. Paris Sér. I. Math. 298 (1984), 217-220.

12. M. G. Crandall, P.-L. Lions and P. E. Souganidis, in preparation.

13. M. G. Crandall and R. Newcomb, Viscosity solutions of Hamilton-Jacobi equations at the boundary, Proc. Amer. Math. Soc. 84 (1985), 283-290.

14. F. Gimbert, Problemes de Neumann quasilineaires, J. Funct. Anal. 62 (1985), 65-72.

15. R. Gonzalez and E. Rofman, On deterministic control problems: an approximation procedure for the optimal cost, Parts I and II, SIAM J. Control Optim. 23 (1985).

16. H. Ishii, On the Perron's method for Hamilton-Jacobi equations, Duke Math. J. 55 (1987), 369-384.

17. __ Uniqueness of unbounded viscosity solutions of Hamilton-Jacobi equations, Indiana Univ. Math. J. 33 (1984), 721-798.

18. __ Remarks on the existence of viscosity solutions of Hamilton-Jacobi equations, Bull. Fac. Sci. Engrg. Chuo Univ. 26 (1983), 5-24.

19. __ Existence and uniqueness of solutions of Hamilton-Jacobi equations, preprint.

20. __ A simple, direct proof of uniqueness for solutions of the Hamilton-Jacobi equations of Eikonal type, preprint.

21. R. Jensen, work in preparation and personal communication.

22. J. M. Lasry and P.-L. Lions, work in preparation; announced in C. R. Acad. Sci. Paris Sér. I. Math. 299 (1987), 213-216.

23. P.-L. Lions, Generalized solutions of Hamilton-Jacobi equations, Pitman, London, 1982; and Existence results for first order Hamilton-Jacobi equations, Richerche Mat. Napoli 32 (1983), 1-23.

24. __ Optimal control and viscosity solutions, Proc. Conf. Dynamic Programming, Rome, 1983, Springer, Berlin, 1985.

25. __ Neumann type boundary conditions for Hamilton-Jacobi equations, Duke Math. J. 52 (1985), 793-820. 
26. __ Quelques remarques sur les problemes elliptiques quasilineaires du second ordre, J. Anal. Math. 45 (1985), 234-254.

27. P.-L. Lions, G. Papanicolau and S. R. S. Varadhan, in preparation.

28. P.-L. Lions and B. Perthame, Quasi-variational inequalities and ergodic impulse control, SIAM J. Control Optim. 24 (1986), 604-615.

29. B. Perthame and R. Sanders, The Neumann problem for fully nonlinear second order singular pertubation problems, M.R.C. Technical Summary Report, Univ. of Wisconsin-Madison, 1986.

30. M. Robin, Long term average cost control problems for continuous time Markov processes: a survey, Acta Appl. Math. (1983).

31. _ On some impulse control problems with long run average cost, SIAM J. Control Optim. 19 (1981), 333-358.

32. M. H. Soner, Optimal control with state-space constraint. I, SIAM. J. Control Optim. 24 (1986).

33. P. E. Souganidis, Existence of viscosity solutions of Hamilton-Jacobi equations, J. Differential Equations (1984).

34. I. Capuzzo-Dolcetta and J. L. Menaldi, On the deterministic optimal stopping time problem in the ergodic case, Theory and Applications of Nonlinear Control Systems (C. I. Byones and A. Lindquist, eds.), North-Holland, Amsterdam, 1986.

Dipartimento di Matematica, Università di Roma-La Sapienza, Piazzale A. Moro 7 , 00100 Roma, ITALY

Ceremade, Université Paris-Dauphine, Place de Lattre de Tasigny, 75775 Paris Cedex 16, France 